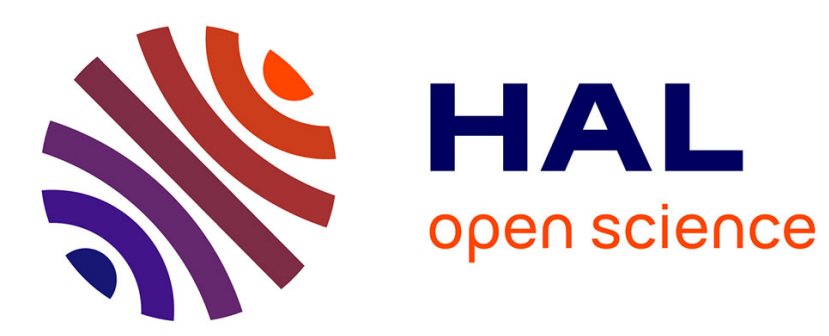

\title{
NMR Studies of the Normal State of High Temperature Superconductors
}

\author{
C. Berthier, M. Julien, M. Horvatić, Y. Berthier
}

\section{To cite this version:}

C. Berthier, M. Julien, M. Horvatić, Y. Berthier. NMR Studies of the Normal State of High Temperature Superconductors. Journal de Physique I, 1996, 6 (12), pp.2205-2236. 10.1051/jp1:1996209 . jpa-00247308

\section{HAL Id: jpa-00247308 https://hal.science/jpa-00247308}

Submitted on 1 Jan 1996

HAL is a multi-disciplinary open access archive for the deposit and dissemination of scientific research documents, whether they are published or not. The documents may come from teaching and research institutions in France or abroad, or from public or private research centers.
L'archive ouverte pluridisciplinaire HAL, est destinée au dépôt et à la diffusion de documents scientifiques de niveau recherche, publiés ou non, émanant des établissements d'enseignement et de recherche français ou étrangers, des laboratoires publics ou privés. 


\title{
NMR Studies of the Normal State of High Temperature Superconductors
}

\author{
C. Berthier $\left({ }^{1.2, *}\right)$, M.H. Julien $\left({ }^{2, *}\right)$, M. Horvatić $\left({ }^{2}\right)$ and Y. Berthier $\left({ }^{1}\right)$ \\ $\left.{ }^{1}\right)$ Laboratoire de Spectrométrie Physique (*), Université Joseph Fourıer, Grenoble I, BP 87 , \\ 38402 Saint-Martin d'Hères Cedex, France \\ $\left({ }^{2}\right)$ Grenoble High Magnetic Field Laboratory, Centre National de la Recherche Scientifique and \\ Max Planck Institut für Festkörperforschung, BP 166X, 38042 Grenoble Cedex 9, France
}

(Received 19 July 1996, recerved in final form 29 August 1996, accepted 30 August 1996)

PACS.74.72.-h - High- $T_{\mathrm{c}}$ compound

PACS.76.60.-k - Nuclear magnetic resonance and relaxation

\begin{abstract}
We review NMR and NQR studies in the normal state of high- $T_{\mathrm{c}}$ superconducting cuprates. Emphasis is given on the three major contributions brought by these techniques: the presence of antiferromagnetic fluctuations, the possible justification of a single spin fluid model, and the opening of a pseudogap in the spin excitations for underdoped compounds. Some recent developments are addressed and compared to theoretical models. In particular, quantitative analysis of the nuclear relaxation are detailed for ${ }^{63} \mathrm{Cu},{ }^{89} \mathrm{Y}$ and ${ }^{17} \mathrm{O}$ nucler, enabling a comparıson with inelastic neutron scatterıng data, and finally pointing out to a serious difficulty with the one-band approach.
\end{abstract}

\section{Introduction}

Besides the appealing perspectives opened by the discovery of a new class of superconductors [1] with values of $T_{c}$ above the temperature of liquid nitrogen, one of the great merit of the discovery of the cuprate high $T_{\mathrm{c}}$ superconductors (HTSC) has been to highlight the peculiar role played by the dimensionality $D=2$ in solid state physıcs. Can one explain the many unconventional properties of the cuprates, including the high value of $T_{c}$, as natural consequences of the presence of Van-Hove singularities in the density of states or antiferromagnetic (AF) fluctuations $[3,4]$. in a quası two-dimensional (2-D) Fermı liquid, or do they require a more fundamental reexammation of low dimensional solid state physics, i.e., the possible extension to 2-D systems of new concepts strictly derıved for one dimensional physıcs [5], like spin-charge separation? Ten years after the discovery of HTSC, these questions are still open, in spite of a huge amount of experımental work involving all techniques avallable in solid state physics. A microscopic description of the quasi-particles present in the normal state $\left(T>T_{\mathrm{c}}\right)$ of these strange metallic compounds is still highly controversial, as is the mechanism responsible for the formation of the superconducting ground state [6]. However, it is generally agreed that the basic ingredient of the $\mathrm{HTSC}$ is $\mathrm{CuO}_{2}$ planes with a square lattice arrangement for $\mathrm{Cu}$

$\left(^{*}\right)$ Authors for correspondence (e-mall: berthi@grenet.fr; mjulıen@polycnrs-gre.fr)

$\left({ }^{*}\right)$ UMR CNRS 5588

(C) Les Éditions de Physıque 1996 


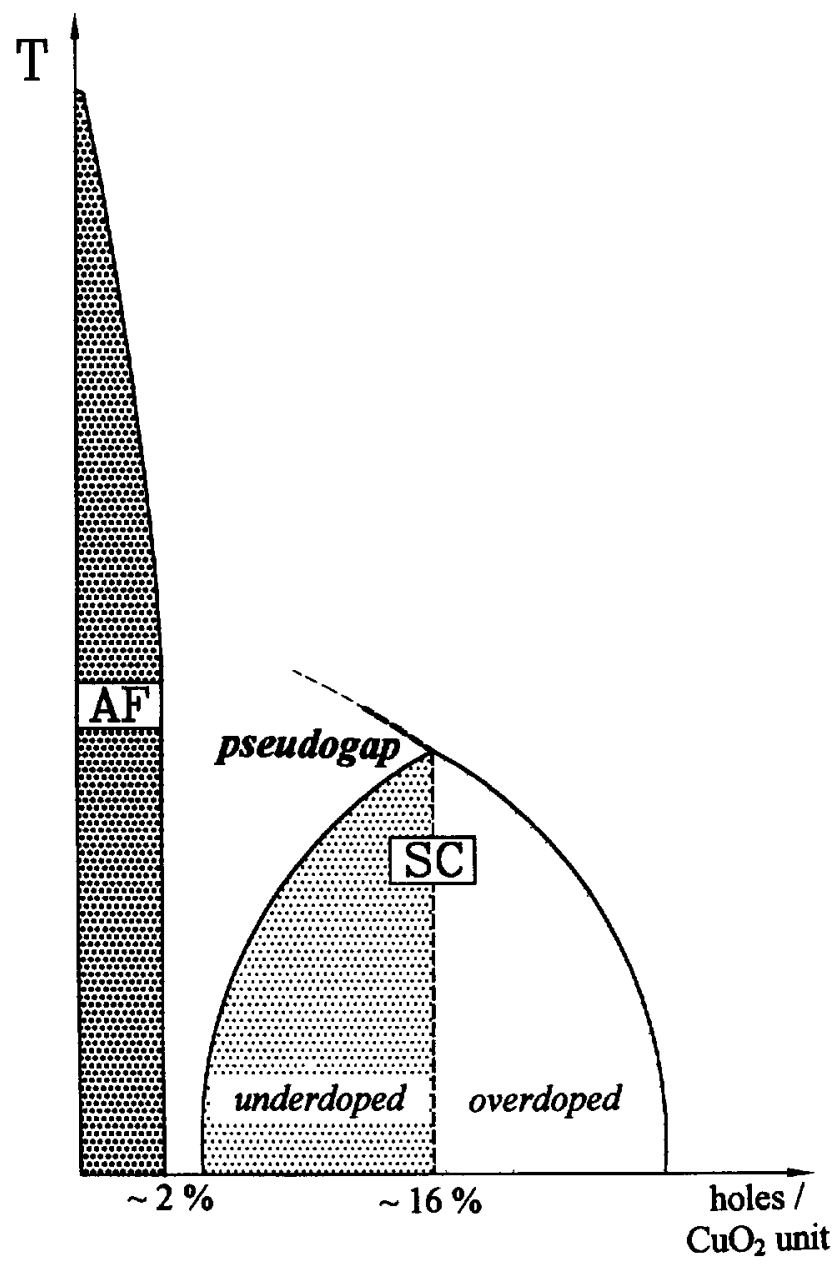

Fig. 1. - Schematic phase diagram for hole doped cuprates. The presence of a pseudogap phase is supported by numerous experıments.

and $\mathrm{O}$ atoms, separated by functional blocks playing the role of a charge reservoir. In the absence of hole (or electron) doping, the $\mathrm{CuO}_{2}$ planes form a good representation of a quasi-2D Heisenberg antiferromagnet $\left(\mathrm{La}_{2} \mathrm{CuO}_{4}, \mathrm{YBa}_{2} \mathrm{Cu}_{3} \mathrm{O}_{6}\right)$ with an insulating Néel ground state. By introducing holes in the $\mathrm{CuO}_{2}$ planes the Néel temperature is rapidly decreased, before producing a new "metallic" phase with a superconducting ground state [7]. The value of the superconducting transition temperature first increases with the hole concentration $n_{\mathrm{h}}$ in the $\mathrm{CuO}_{2}$ plane (underdoped regime), reaches a maximum value for an optimal doping, and then decreases with a further increase of $n_{\mathrm{h}}$ (overdoped regime) [8]. The full evolution according to this ideal phase diagram (Fig. 1) is not usually observed in a single system. For example, a variation in $x$ going from 0 to 1 in $\mathrm{YBa}_{2} \mathrm{Cu}_{3} \mathrm{O}_{6+v}$ shows an $\mathrm{AF}$ phase for $x<0.4$, covers the underdoped regime from 0.4 to $0.94\left(T_{\mathrm{c}}=93 \mathrm{~K}\right)$, and has a small range of concentration in the overdoped regime. $\mathrm{La}_{2-x} \mathrm{Sr}_{x} \mathrm{CuO}_{4}$ as a function of $x$ exhibits first an AF phase, separated from the metallic one by a spin-glass regime. The optimal doping is around $x=n_{\mathrm{h}}=0.15$ and the 
overdoped range is difficult to obtain in stable form [9]. On the contrary, $\mathrm{Tl}_{2} \mathrm{Ba}_{2} \mathrm{CuO}_{6+\delta}$ only exists in the overdoped regime, with $T_{\mathrm{c}}$ values ranging between $\approx 85$ to $0 \mathrm{~K}$. Nowadays, there $1 \mathrm{~s}$ more and more experimental evidence that the underdoped and the overdoped regimes have quite different electronic properties [10-12]. Indeed, this was inferred very early [13,14], from the experimental data obtained by Nuclear Magnetic Resonance (NMR) in various HTSC systems. This technique, which played a crucial role in the test of the Bardeen-Cooper-Shrieffer (BCS) theory for conventional superconductors $[15,16]$, has been extensively applied to the study of the HTSC during the past decade and has proved to be a major tool for investigating the low energy excitations in these compounds.

The goal of this paper is to review the current knowledge of NMR experimental data in the cuprates and to discuss how they put constraints on the various theoretical models competing for the description of the quasi-particles which are responsible for the superconducting ground state. We shall limit ourselves to the normal state $\left(T>T_{c}\right)$ of the metallic part of the phase diagram. For NMR studies of the antiferromagnetic phase, and that of the superconducting state, we refer the reader to other review papers [17-22]. The paper will be organized as follows: in Section 2, we review the NMR background necessary to appreciate the contribution of NMR to our better understanding of the physical properties of the cuprates. Section 3 will be devoted to the notion of single spin fluid, which has been supported by NMR, to the Mila-Rice Hamitonian commonly used to describe the Hamiltonian of the various nuclei in the cuprates, and to the description of the magnetic hyperfine couplings. In Section 4, we review the experimental results obtained by nuclear spin lattice and spin-spin relaxation measurements, and discuss current interpretations in the literature, within the framework of the nearly antiferromagnetic Fermı liquid, and the quantum critical regime of disordered 2D Heisenberg antiferromagnets. Section 5 is devoted entirely to the question of the spin gap in underdoped systems, which was first revealed by NMR experiments, and has become a central issue in the understanding of electronic and magnetic properties of the normal state of HTSC. In Section 6, we discuss the comparison between NMR and inelastic neutron scattering data and how it affects the interpretation of the NMR data. Section 7 briefly mentions the NMR data concerning the problems of phase separation. In the concluding remarks, we try to summarize the contribution of NMR to the present understanding of the properties of the normal state of HTSC, and to stress upon the open questions which should be addressed in a near future.

\section{NMR and NQR Background}

Nuclear spins in solids are submitted to various couplings due to their environment, formed by localized and delocalized electrons in the structure. Their total Hamiltonian can be written as:

$$
\mathcal{H}_{\text {total }}=\mathcal{H}_{\text {zeeman }}{ }^{\prime}+\mathcal{H}_{\mathrm{Q}}+\mathcal{H}_{\text {mhf }}+\mathcal{H}_{\text {nucle1-nucle1 }}
$$

where the various terms are respectively the Zeeman term. the quadrupolar term, the magnetic hyperfine interaction, and the nuclear spin-spin interaction.

NMR usually refers to the case where the main Hamiltonian is the Zeeman coupling of the nuclear spins $\mathbf{I}$ to the external magnetic field $\mathbf{H}=H_{0} \mathbf{k}, \mathcal{H}_{\text {zeeman }}=-\gamma_{2} \hbar \sum_{2} I_{z}^{2} H_{0}$, where $\gamma_{2}$ is the gyromagnetic ratio of the isotope $i$. In the absence of any other perturbation, the Zeeman Hamiltonian gives rise to a single line spectrum, at the Larmor frequency $\omega_{\mathrm{n}}=2 \pi \gamma_{2} H_{0}$.

2.1. Electric Field Gradients. - For spin $I>1 / 2$, and non-cubic local symmetry, the largest perturbation to the Zeeman Hamiltonian is usually the quadrupole Hamiltonian $\mathcal{H}_{Q}$, 
which couples the quadrupole moment $Q$ of the nuclei to the electric field gradient (EFG) due to the surrounding electronic charge distribution. Note that $Q$ is specific to each isotope. The EFG tensor is a second rank tensor which usually strongly reflects the local symmetry of the environment. If we call $V_{\alpha \alpha}$ the components of the EFG tensor along its principal axes $(\alpha=X, Y, Z), \mathcal{H}_{\mathrm{Q}}$ can be expressed as:

$$
\mathcal{H}_{\mathrm{Q}}=\frac{h \nu_{\mathrm{Q}}}{2}\left[I_{Z}^{2}-\frac{I(I+1)}{3}+\frac{\eta}{6}\left(I_{+}^{2}+I_{-}^{2}\right)\right]
$$

where $\nu_{\mathrm{Q}}$ is called the quadrupole frequency, $\eta=\frac{V_{X X}-V_{Y Y}}{V_{Z Z}}$ is the asymmetry parameter with the convention $\left|V_{Z Z}\right| \geq\left|V_{Y Y}\right| \geq\left|V_{X X}\right|$, and $\nu_{\mathrm{Q}}$ is related to the largest component of the $\mathrm{EFG}$ tensor by $\nu_{\mathrm{Q}}=\frac{3 e Q V_{Z Z}}{2 I(2 I-1) h}$. In a high magnetic field, the quadrupole perturbation (for odd values of $2 I)$ leads to a splitting of the resonance into a central line $(m=-1 / 2 \leftrightarrow 1 / 2$ transition) and satellites on each side of it, the value of the splitting being related to both $\nu_{\mathrm{Q}}$ and the orientation of the external magnetic field with respect to the principal axis of the EFG tensor. In the case where $\nu_{\mathrm{Q}}$ is large, the spectrum for unoriented powder samples is a complicated pattern $[23,24]$, which usually inhibits useful studies. Fortunately, most of the cuprate powder samples can be oriented with the application of an external magnetic field, so that the $c$-axis (perpendicular to the $\mathrm{CuO}_{2}$ plane) of each of the grains (which have to be single-crystals) is aligned along the field direction [25]. Working on such oriented powders with $H_{0} / / c$-axis is equivalent to work on single crystals. When $H_{0}$ is perpendicular to the $c$-axis, two different situations are encountered: if there is no detectable asymmetry of the tensor in the $a b$-plane (which is the case for the planar copper) the spectrum is again similar to that obtained in a single crystal. However, when the largest component of the tensor lies in the $a b$-plane (copper in the chains of $\mathrm{YBa}_{2} \mathrm{Cu}_{3} \mathrm{O}_{6+x}$, planar oxygen) one obtains a broad $2 \mathrm{D}$ powder spectrum.

In the absence of both external magnetic field and spontaneous spin polarization, $\mathcal{H}_{\mathrm{Q}}$ becomes the main Hamltonian and transitions can be induced between its eigenstates. This situation is called Nuclear Quadrupole Resonance (NQR), a technique which has been widely used in the study of cuprates, namely for $\mathrm{Cu}$ and La nuclei (the quadrupole coupling at the oxygen sites is too small to allow a direct detection in zero external magnetic field). One advantage of NQR is the possibility to follow the nuclear spin-lattice relaxation time $T_{1}$ in zero field, avoiding complications related to the presence of vortices in the mixed phase.

The knowledge of the quadrupole tensor for each particular nuclear site (when it exists) 1s a prerequisite to study the $\mathcal{H}_{\text {mhf }}$ Hamiltonian. Studies of the EFG tensor provide valuable information about the site symmetry and the degree of disorder in the local environment [26.27]. A recent development is worth being noted: thanks to the increasing number of NMR studies In various systems, it seems that all compounds having a high (or potentially high) $T_{\mathrm{c}}$ show systematically relatively low $\nu_{\mathrm{Q}}$ values for $\mathrm{Cu}$ in the $\mathrm{CuO}_{2}$ planes. This behavior, first noted by Zheng et al. [28], has been tentatively ascribed to a higher in-plane $\mathrm{Cu}-\mathrm{O}$ hybridization. Such a conclusion seems to be supported by recent NMR results in Hg-based cuprates where the buckling of the $\mathrm{CuO}_{2}$ planes is negligible $[29,30]$. However, the respective werghts of the electronic on-site and lattice contributions to the EFG are not well understood, making this issue still controversial.We shall not discuss the EFG tensors in more detail here; the reader is referred to review [19] or specialized papers [31,32], dealing with this matter.

2.2. Magnetic Hyperfine Shifts. - Here, we shall concentrate on the magnetic hyperfine interaction. The net result of the Hamiltonian $\mathcal{H}_{\text {mhf }}$ can be considered as the interaction of a 
nuclear spin with a time dependent local magnetic hyperfine field $h_{\mathrm{L}}$ due to the electronic spins and orbital motion. The static (time-averaged) part $h_{\mathrm{L}}$ gives rise to a shift of the NMR line proportional to the applied external field, which is denoted as the Magnetic Hyperfine Shift (MHS) tensor K. Its components can be decomposed into a spin, an orbital and a diamagnetic part:

$$
K_{\alpha \alpha}(T)=K_{\alpha \alpha}^{\text {spin }}+K_{\alpha \alpha}^{\text {orb }}+K_{\alpha \alpha}^{\text {dia }}
$$

where $\alpha$ denotes the $x, y, z$ directions. In HTSC compounds, $\mathbf{K}^{\text {orb }}$ is temperature independent and $\mathbf{K}^{\text {dia }}$ is usually negligible. Well below $T_{\mathrm{c}}, \mathbf{K}^{\text {spin }}$ vanishes, as the signature of singlet spin pairing and thus, the remaining MHS tensor is the orbital one. In the following, we shall only consider the MHS tensor of the copper and oxygen nuclei in the $\mathrm{CuO}_{2}$ plane (usually referred to as $\mathrm{Cu}(2)$ and $\mathrm{O}(2,3)$ in the case of $\left.\mathrm{YBa}_{2} \mathrm{Cu}_{3} \mathrm{O}_{6+x}\right)$, and that of the yttrium when present in between the $\mathrm{CuO}_{2}$ planes. (In all NMR studies, the apex oxygen nuclei have been found to be quite weakly coupled to the spin susceptibility, and therefore results concerning this site will not be discussed here.) For copper and yttrium, the MHS tensors have an axial symmetry and the components will be denoted $K_{c}$ and $K_{a b}$ for $H_{0}$ parallel or perpendicular to the $\mathrm{CuO}_{2}$ plane [33]. For the planar oxygen sites, the main component of the MHS tensor lies along the $\mathrm{Cu}-\mathrm{O}-\mathrm{Cu}$ bond; thus the in-plane components are denoted as $K_{/ /}$along this bond and $K_{\perp}$ perpendicular to it, and the MHS tensor is usually expressed as $\left(K_{c}^{-}, K_{\perp}\right.$ and $\left.K_{/ /}\right)$.

The spin part of the MHS tensor can be formally related to the average susceptibility per site through the relationship:

$$
K_{\alpha \alpha}=A_{\alpha \alpha} \chi_{\alpha \alpha} / g_{\alpha} \mu_{\mathrm{B}}
$$

where $A_{\alpha \alpha}$ is the hyperfine field per spin unit [34], $g_{\alpha}$ is the Lande factor for the electron, $\mu_{\mathrm{B}}$ the Bohr magneton, and $\alpha$ is the direction of the magnetic field, which we always suppose to be along one of the principal axis of the MHS tensor. This simple formula hides actually a rather complex reality. One ambiguity in the cuprates is whether one should consider the electronic spins as localized as in magnetic insulators [35] or as itinerant as in transition metals for example [36]. In both cases, the coupling between the nucleus and the electronic spin density is rather local, and usually decomposed in several components corresponding to the spin polarization of the various atomic wave functions on which the whole many body electronic wavefunction is projected. For example, in the case of the planar $\mathrm{Cu}$ site, the spin part of the MHS is expected to be mainly due to the spin polarızation of the on-site $\mathrm{d}_{x^{2}-y^{2}}$ orbital. However, after an analysis of the experimental MHS tensor [37,38], of the planar copper in $\mathrm{YBa}_{2} \mathrm{Cu}_{3} \mathrm{O}_{7}$, it was concluded that an important contribution was due to a super-transferred hyperfine field proportional to the spin polarization carried by the nearest neighbors $\mathrm{Cu}(2)$. through the oxygen $\mathrm{O}(2 \mathrm{p})$ and the $\mathrm{Cu}(4 \mathrm{~s})$ wave-functions [39]. As far as the MHS tensor of the planar oxygen is concerned, it has to be analyzed in terms of spin polarization of the oxygen $\mathrm{O}(2 \mathrm{p})$ and $\mathrm{O}(2 \mathrm{~s})$ orbitals [40-42]. Consequently, after removal of the dipolar contribution due to the spin density on the $\mathrm{Cu}(2)$ sites, the MHS tensor of oxygen is written as:

$$
{ }^{17} K^{\text {spin }}(\theta)={ }^{17} K_{\text {sso }}^{\text {spin }}+{ }^{17} K_{\mathrm{ax}}^{\text {spin }} \frac{\left(3 \cos ^{2} \theta-1\right)}{2}
$$

where ${ }^{17} K_{\text {iso }}$ and ${ }^{17} K_{\text {ax }}$ are related to the spin polarızation of the $\mathrm{O}(2 \mathrm{~s})$ and $\mathrm{O}(2 \mathrm{p})$ orbitals, respectively, and $\theta$ is the angle between the magnetic field $H_{0}$ and the $\mathrm{Cu}-\mathrm{O}-\mathrm{Cu}$ bond in the $a b$-plane. In equation (5) we neglected the small deviation from the axial symmetry which is revealed by experımental data, so that experimentally, ${ }^{17} K_{\text {iso }}$ and ${ }^{17} K_{\text {ax }}$ are defined as:

$$
{ }^{17} K_{\text {1so }}=\left({ }^{17} K_{\mathrm{c}}+{ }^{17} K_{\perp}+{ }^{17} K_{/ /}\right) / 3,{ }^{17} K_{\mathrm{ax}}=2\left[{ }^{17} K_{/ /}-\left({ }^{17} K_{\mathrm{c}}+{ }^{17} K_{\perp}\right) / 2\right] / 3
$$


Finally, the yttrium MHS tensor is dominated by the core-polarization of closed yttrium shells by the spin density at the $\mathrm{Cu}(2)$ site through the $\mathrm{O}(2 \mathrm{p})$ orbitals [43]. Similar situation can be found for other nucle1 like ${ }^{43} \mathrm{Ca},{ }^{205} \mathrm{Tl}$, or ${ }^{199} \mathrm{Hg}$ in other systems, but we shall not comment on them here.

2.3. Nuclear Spin Lattice Relaxation. - The nuclear spin-lattice relaxation rate (NSL $\mathrm{RR}$ ), which is the rate at which the nuclear magnetization relaxes to its thermal equilibrium value along the external magnetic field, is due to the fluctuations of the magnetic hyperfine field $h_{\mathbf{L}}$ (the fluctuations of the EFG tensor have been shown to have a negligible contribution in the normal state, at least for the nuclei species which have been studied). According to Moriya [44], the NSLRR rate can be written as:

$$
{ }^{\imath}\left(T_{1} T\right)_{\alpha}^{-1}=\frac{k_{\mathrm{B}} \gamma_{2}^{2}}{\mu_{\mathrm{B}}^{2}} \sum_{q, \alpha^{\prime} \neq \alpha}\left|A_{\alpha^{\prime} \alpha^{\prime}}^{2}(q)\right|^{2} \frac{\chi_{\alpha^{\prime} \alpha^{\prime}}^{\prime \prime}\left(q, \omega_{\mathrm{n}}\right)}{g_{\alpha^{\prime} \alpha^{\prime}}^{2} \omega_{\mathrm{n}}}
$$

where $\imath$ stands for the nuclear species, $\alpha$ for the direction of $H_{0}$ (supposed to be parallel to one of the principal axes of $A_{\alpha^{\prime} \alpha^{\prime}}$ and $\chi_{\alpha^{\prime} \alpha^{\prime}}^{\prime \prime}$ tensors $), A_{\alpha^{\prime} \alpha^{\prime}}(q)$ is the Fourier transform of the hyperfine field defined above $\left[A_{\alpha^{\prime} \alpha^{\prime}}(\mathbf{q})=\sum_{R \imath} A_{\alpha^{\prime} \alpha^{\prime}}\left(\mathbf{R}_{2}\right) \exp \left(-i \mathbf{q} \cdot \mathbf{R}_{2}\right)\right]$, and $\chi_{\alpha^{\prime} \alpha^{\prime}}^{\prime \prime}\left(q, \omega_{n}\right)$ is the imaginary part of dynamic spin susceptibility for the wavevector $\mathbf{q}$ and nuclear Larmor frequency $\omega_{\mathrm{n}}\left(\hbar \omega_{\mathrm{n}} \approx 0\right)$ for the direction $\alpha^{\prime}$ perpendicular to $\alpha$. Again, the use of this formula in HTSC compounds hides some ambiguity, depending on whether one considers the electronic spins as localized or itinerant. For example, in the case of the oxygen relaxation. the use of the traditional formulae for $\left(T_{1} T\right)^{-1}$ in metals $[35,36]$ would lead to consider the fluctuations of the isotropic part of the hyperfine tensor and that of the axial one, as incoherent. On the contrary, in the picture of localized spins at the copper site, coupled to the oxygen nuclei through a transferred hyperfine field, $A_{1 \text { so }}$ and $A_{\mathrm{ax}}$ act coherently. Obviously, both descriptions lead to quite different values for the anisotropy of the oxygen NSLRR. Experimental data [45, 46]. favor the localized picture.

2.4. Nuclear Spin-Spin Relaxation. - In most solıds, the Hamiltonian $\mathcal{H}_{\text {nuclej-nucle }}$ is restricted to the nuclear dipole-dipole interaction. It thus gives rise to a temperature independent spin-spin relaxation rate which often has the form $\exp \left(-t^{2} / 2\left(T_{2 \mathrm{G}}\right)^{2}\right)$ where $1 /\left(T_{2 \mathrm{G}}\right)^{2}$ is equal to the second-moment of the line-shape. By designating $H_{\mathrm{nn}}^{\prime}$ as the part of $\mathcal{H}_{\text {nucle-nucles }}$ which connects states of the same energy, $1 /\left(T_{2 \mathrm{G}}\right)^{2}$ can be evaluated as a trace:

$$
1 /\left(T_{2 \mathrm{G}}\right)^{2}=-\frac{\operatorname{Tr}\left[H_{\mathrm{nn}}^{\prime}, I_{x}\right]^{2}}{\hbar^{2} \operatorname{Tr} I_{x}^{2}}
$$

However, indirect nuclear spin-spin interactions can be mediated by the electronıc spin susceptibility [36]. The group of Slichter [47] was the first to point out the importance of $T_{2 \mathrm{G}}$ to obtain information about the antiferromagnetic exchange between the electronic spins. Formally, this type of indirect spin-spin interaction can be written as:

$$
\sum_{\alpha, 2,3} \frac{\gamma_{\imath} \gamma_{\jmath}}{2\left(g_{\alpha \alpha} \mu_{\mathrm{B}}\right)^{2}} I_{\alpha}^{2} I_{\alpha}^{3} \sum_{R, R^{\prime}} A_{\alpha \alpha}\left(R_{2}-R\right)_{\lambda}\left(R-R^{\prime}\right) A_{\alpha \alpha}\left(R_{\jmath}-R^{\prime}\right)
$$

where $\chi\left(\mathbf{R}-\mathbf{R}^{\prime}\right)$ is the Fourler transform of the real part of the electronic spin susceptibility $\chi(\mathbf{q})$. Later, it has been shown that this quantity was temperature dependent $[48,49]$, and that the nuclear dipole-dipole contribution was negligible compared to that of the indirect 
interactions. The study of the temperature dependence of $T_{2 \mathrm{G}}$ has become a major tool in NMR and NQR studies of HTSC, since due to the presence of a transferred hyperfine interaction in the these materials (see Sect. 3.2.), $T_{2 \mathrm{G}}$ is able to provide information about the static part of the staggered susceptibility $\chi^{\prime}\left(\mathbf{q} \approx \mathbf{Q}_{\mathrm{AF}}, \omega=0\right)$.

\section{Magnetic Hyperfine Couplings}

3.1. A Single Spin-Fluid. - In the first few years after the discovery of the cuprates, there has been an intense effort to find the simplest model Hamiltonian which would describe the low energy properties of the $\mathrm{CuO}_{2}$ planes. Very early, Anderson [5] proposed an effective Hamiltonian known as the t-J model, which corresponds to a single band 2D Hubbard model in the limit of infinite on-site Coulomb repulsion $U$. Whether such a simple model could keep the essential features of a more general Hamltonian containing $\mathrm{Cu}$ and $\mathrm{O}$ bands and intra- and interatomic Coulomb interactions has been a long debate. To justify the t-J model, Zhang and Rice [50] have proposed that the holes introduced by doping the $\mathrm{CuO}_{2}$ planes would delocalize onto the four oxygen sites around a $\mathrm{Cu}^{2+}$ ion and form a local singlet with 1t. This singlet would then move through the lattice of $\mathrm{Cu}^{2+}$ ions, $\mathrm{m}$ a similar way as a hole. i.e., a missing spin, in a simple square $\mathrm{Cu}^{2+}$ ion lattice. The ability of the $\mathrm{t}-\mathrm{J}$ model to cover the whole physics of the starting two-band Hamiltonian has been criticized by Emery and Reiter [51], who claimed that the two-band Hamiltonian implies the existence of a second spin degree of freedom related to the $O(2 p)$ band, which cannot be accounted within the framework of the t-J model. This question has polarized the interpretation of the temperature dependence of the magnetic hyperfine shifts in high $T_{\mathrm{c}}$ superconductors. Early measurements of the anisotropic part of the MHS at oxygen sites in $\mathrm{YBa}_{2} \mathrm{Cu}_{3} \mathrm{O}_{7}$ [40-42], which is directly related to the spin polarization of the $\mathrm{O}(2 \mathrm{p})$ orbitals, have shown that most of the total spin polarization $(>85 \%$ ) was carried by the copper sites in these compounds. As regards the temperature dependence of the MHS, it was first reported by Alloul et al. [52] that in the $\mathrm{YBa}_{2} \mathrm{Cu}_{3} \mathrm{O}_{6+x}$ system, the MHS of yttrium was scaling linearly the macroscopic susceptibility. Since the main coupling of the yttrium to the magnetic susceptibility in the $\mathrm{CuO}_{2}$ plane occurs through the polarization of the oxygen orbitals this was interpreted as a proof of the existence of a single spin fluid [53] in agreement with the t-J model. Soon after, it was shown by Takigawa et al. [54] that ${ }^{63} K_{a b},{ }^{17} K_{\text {iso }}$, and ${ }^{17} K_{\text {ax }}$ had the same temperature dependence in $\mathrm{YBa}_{2} \mathrm{Cu}_{3} \mathrm{O}_{6.63}$ (Fig. 2). Subsequently, this behavior, i.e., the same $T$-dependence on all nuclear sites coupled to the $\mathrm{Cu}$ spin polarization, was observed in all the cuprates where it was studied ( $\mathrm{YBa}_{2} \mathrm{Cu}_{4} \mathrm{O}_{8}$ [55], $\mathrm{La}_{2-x} \mathrm{Sr}_{x} \mathrm{CuO}_{4}$ [56] for example). Strictly speaking, this behavior by itself does not exclude the existence of a temperature independent susceptibility due to an $\mathrm{O}(2 \mathrm{p})$ band. For example, the $T$ dependence of the Knight shift in Platinum metal varies linearly with the macroscopic susceptibility. regardless of the presence of an s-p band giving rise to a $T$ independent contribution [57]. The validity of the single spin-fluid picture in HTSC thus relies on the precise knowledge of the orbital shifts of the oxygen, copper, and yttrium nuclei. Let us consider for example the axial part of the oxygen MHS. There are three possible contributions: one proportional to the polarization of the $\mathrm{O}(2 \mathrm{p})$ orbitals by hybridization with the $\mathrm{Cu}(2)$ orbitals, one proportional to the susceptibility (supposed to be temperature independent) of some $O(2 p)$ band, and a (temperature independent) orbital contribution:

$$
{ }^{17} K_{\mathrm{ax}}(T)-{ }^{17} K_{\text {const }}=2 f_{\mathrm{p}} A^{2 \mathrm{p}} \chi^{\mathrm{spin}}=\lambda\left({ }^{63} K_{a b}(T)-{ }^{63} K_{\text {orb }}\right) \quad T>T_{\mathrm{c}}
$$

with

$$
{ }^{17} K_{\text {const }}=A^{2 \mathrm{p}} \chi^{\text {holes }}+{ }^{17} K_{\mathrm{ax}}^{\text {orb }}
$$




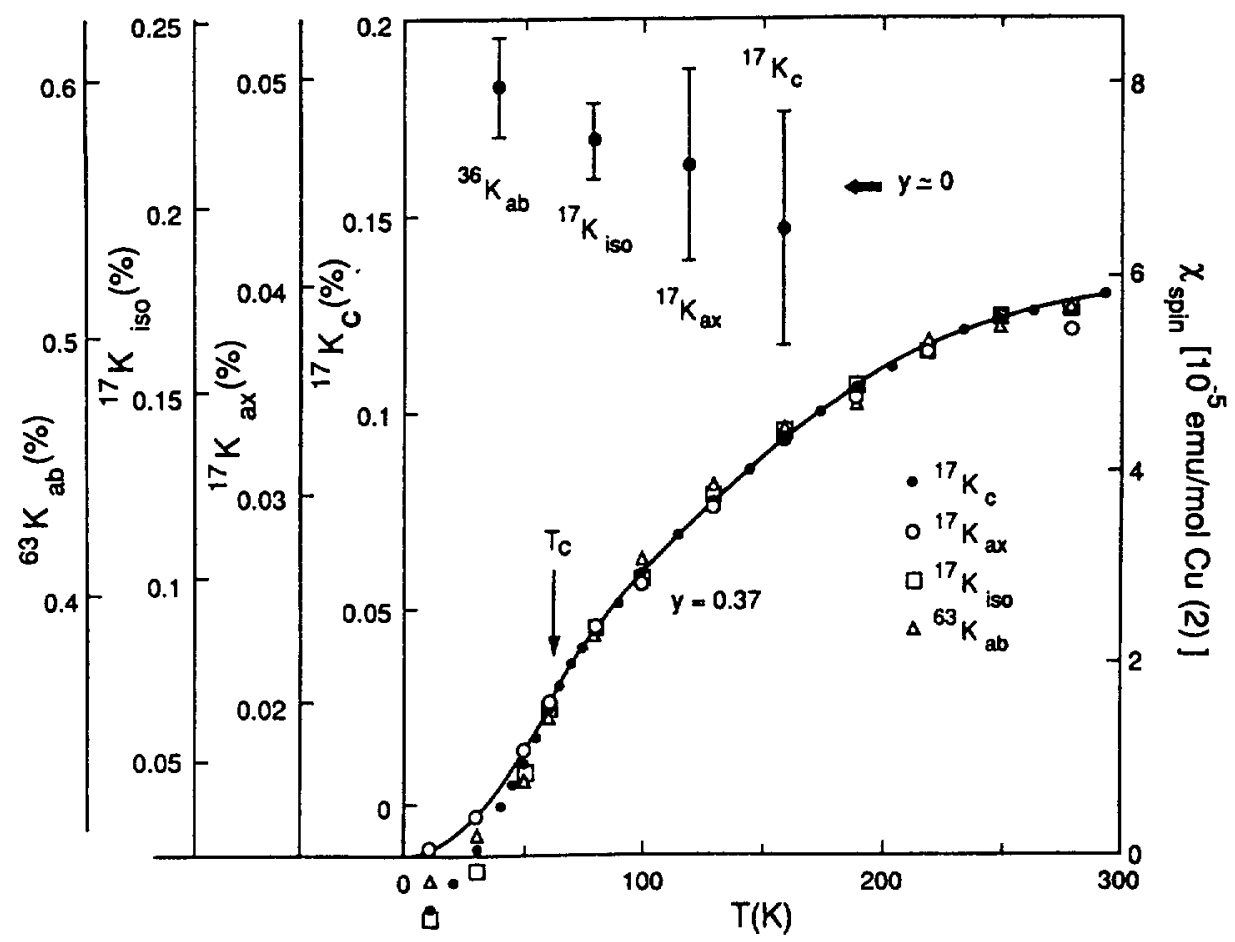

Fig. 2. - Various components of the $\mathrm{Cu}$ and $\mathrm{O}$ magnetic hyperfine shift tensors in $\mathrm{YBa}_{2} \mathrm{Cu}_{3} \mathrm{O}_{6} 63$. From Takigawa et al. [54].

$$
{ }^{17} K_{\mathrm{ax}}(T=0)={ }^{17} K_{\mathrm{ax}}^{\mathrm{rorb}} \quad{ }^{63} h_{\mathrm{ax}}(T=0)={ }^{63} K_{\text {orb }} \quad T \ll T_{\mathrm{c}}
$$

It is clear that the single spin fluid hypothesis can only be validated if experimentally, ${ }^{17} K_{\text {const }}=$ ${ }^{17} K_{\mathrm{ax}}^{\mathrm{orb}}$. It can be seen in reference [54] that although some deviation from this equality seems to occur, it is well within the error bars. and leads to a negligible value of $\chi^{\text {holes }}$ However, one must remember that the determination of the orbital shifts in the superconducting state is difficult. because of the corrections due to the finite magnetization in the mixed state and the change in the lineshape due to the presence of a vortex lattice [16].

3.2. The Mila-Rice Hamiltonian, Hyperfine Coupling Tensors and Form FactORS. - The single spin fluid picture, and the fact that most of the spin density is borne by the copper sites, has led to the following hyperfine Hamiltonian, in which the only source of coupling are the electronic spins localized at the copper site:

$\mathcal{H}_{\mathrm{mhf}}={ }^{63} \gamma \hbar \sum_{\imath}{ }^{63} \mathbf{I}_{2}\left(\mathbf{A} \mathbf{S}_{\imath}+\sum_{\delta=1-4} B \mathbf{S}_{\imath+\delta}\right)+{ }^{17} \gamma \hbar \sum_{\jmath, \delta^{\prime}=1-2}{ }^{17} \mathbf{I}_{\jmath} \mathbf{C S}_{\jmath+\delta^{\prime}}+{ }^{89} \gamma \hbar_{k} \sum_{k, \delta^{\prime \prime}=1-8}{ }^{89} \mathbf{I}_{k} \mathbf{D} \mathbf{S}_{k+\delta^{\prime \prime}}$

where the tensor $\mathbf{A}$ corresponds to the $\mathrm{Cu}$ on-site hyperfine field tensor, $B$ is the (isotropic) supertransfered hyperfine field from the 4 nearest neighbors copper sites, $\mathbf{C}$ is the (ansotropic) transferred hyperfine coupling to the planar oxygen from its two nearest neighbors copper sites, and $\mathbf{D}$ the supertransfered hyperfine tensor to the yttrium from the eight nearest neighbors copper sites (in the case of $\mathrm{YBa}_{2} \mathrm{Cu}_{3} \mathrm{O}_{6+x}$ and related compounds). This Hamiltonian, usually quoted as the Mila-Rice or the Shastry-Mila Rice Hamiltonian [58] is commonly used in the 
literature to quantitatively analyze the NMR data. The first step in the analysis of NMR data usually concerns the determination of the hyperfine field. However, this is not obvious in the cuprates for several reasons. In the case of the planar copper, there are three quantities to determine: $A_{c}, A_{a b}$ and $B$, although experimentally, only $A_{c}+4 B$ and $A_{a b}+4 B$ can be obtained from equation (4). The determination of $A_{c}+4 B, A_{a b}+4 B, C_{\alpha}$, and $D_{\alpha}$ is more accurate in compounds where the susceptibility is strongly temperature dependent, that 1s, in underdoped compounds. Indeed, few experiments that include susceptibility and MHS measurements on the same samples can be found in the literature $[42,56,59,60]$. In addition, it is not obvious what susceptibility to use in equation (4) in compounds like $\mathrm{YBa}_{2} \mathrm{Cu}_{3} \mathrm{O}_{6+x}$ where part of the susceptibility is carried by the copper atoms located on the chains. Usually, the $\mathrm{Cu}$ atomic susceptibility is obtained by dividing the total susceptibility by $2+x$ and assuming that copper in the chains and in the planes bear the same spin susceptibility. This assumption has been used as well for the determination of $A_{a b}+4 B$ [59] and $C_{\alpha \alpha}$ [42]. The anisotropy of the static susceptibility is usually not taken into account. As far as $A_{c}+4 B$ is concerned, it turns out that experimentally for $H_{0} / / c$-axis, the MHS is found independent of temperature and have identical values in the metallic and superconducting state within the experimental uncertainties in the $\mathrm{YBa}_{2} \mathrm{Cu}_{3} \mathrm{O}_{6+x}, \mathrm{YBa}_{2} \mathrm{Cu}_{4} \mathrm{O}_{8}, \mathrm{La}_{2-x} \mathrm{Sr}_{x} \mathrm{Cu}_{2} \mathrm{O}_{4}$ compounds. Thus $A_{c}+4 B \approx 0$ is an usual assumption in all these compounds. The same is not true for compounds like $\mathrm{Tl}_{2} \mathrm{Ba}_{2} \mathrm{CuO}_{6+\delta}$ [61], $\mathrm{Tl}_{2} \mathrm{Ba}_{2} \mathrm{Ca}_{2} \mathrm{Cu}_{3} \mathrm{O}_{10}$ [62], $\mathrm{Bi}_{2} \mathrm{Sr}_{2} \mathrm{CaCu}_{2} \mathrm{O}_{8}$ [63, 64], and $\mathrm{HgBa}_{2} \mathrm{Ca}_{2} \mathrm{Cu}_{3} \mathrm{O}_{8}[29,30]$. By assuming that $A_{c}$ and $A_{a b}$ are intrinsic properties of the planar $\mathrm{Cu}$ ion and taking the current values for $\mathrm{YBa}_{2} \mathrm{Cu}_{3} \mathrm{O}_{6+x}$, tentative values of $B$ have been extracted from the ratio $\left(A_{c}+4 B\right) /\left(A_{a b}+4 B\right)$. They are systematically larger than in the YBCO and LASCO compounds, which may indicate a larger value of the exchange coupling in these materials $[29,30,61,62]$. This behavior is usually accompanied by a smaller value of the planar copper quadrupole coupling, as already mentioned above, a feature which has been attributed to a larger hybridization between $d_{x^{2}-y^{2}}$ and $\mathrm{O}(2 \mathrm{p})$ orbitals $[28,29]$. Both phenomena seem to be controlled by the buckling of the $\mathrm{Cu}-\mathrm{O}-\mathrm{Cu}$ bonding.

Finally, the ratio $2 C_{c} /\left(A_{a b}+4 B\right)$ has been determined in some compounds $\mathrm{YBa}_{2} \mathrm{Cu}_{3} \mathrm{O}_{6.63}$ (0.58) [54], $\mathrm{YBa}_{2} \mathrm{Cu}_{4} \mathrm{O}_{8}$ (0.67) [55], $\mathrm{La}_{2-x} \mathrm{Sr}_{x} \mathrm{CuO}_{4}$ (0.68) [56], $\mathrm{Tl}_{2} \mathrm{Ba}_{2} \mathrm{CuO}_{6+\delta}$ (0.30) [61], $\mathrm{Tl}_{2} \mathrm{Ba}_{2} \mathrm{Ca}_{2} \mathrm{Cu}_{3} \mathrm{O}_{10}(0.50)$ [62] as well as $\left(8 D_{\mathrm{c}}+D_{\mathrm{dip}_{\mathrm{c}}}\right) / C_{\mathrm{c}}(-0.127)$ in $\mathrm{YBa}_{2} \mathrm{Cu}_{3} \mathrm{O}_{663}$ [65]. Typical values of the hyperfine field constants can be found in references $[22,66,67]$. It must be emphasized that these values are approximate: in the most favorable cases they are not known with an accuracy better than $20 \%$.

The knowledge of these coupling is of primary importance for a quantitative interpretation of the nuclear spin-lattice and spin-spin relaxation data. In the hypothesis of a single spin degree of freedom, the evaluation of $1 / T_{1}$ and $1 / T_{2 \mathrm{G}}$ involves a summation over the Brillouin zone of the product of the imaginary or the real part of the dynamic spin susceptibility located at the planar copper sites square with the form factors $F(\mathbf{q})=|A(\mathbf{q})|^{2}$ These form factors $F(q)$ can be expressed as:

$$
\begin{aligned}
{ }^{63} F_{a b}(\mathbf{q}) & =\left[A_{a b}+2 B\left(\cos q_{x} a+\cos q_{y} b\right)\right]^{2} \\
{ }^{63} F_{c}(\mathbf{q}) & =\left[A_{c}+2 B\left(\cos q_{x} a+\cos q_{y} b\right)\right]^{2} \\
{ }^{17} F_{\alpha}(\mathbf{q}) & =2 C_{\alpha \alpha}^{2}\left[1+\left(\cos q_{x} a+\cos q_{y} b\right) / 2\right] \\
{ }^{89} F_{\alpha}(\mathbf{q}) & =8 D_{\alpha a}^{2}\left(1+\cos q_{x} a\right)\left(1+\cos q_{y} b\right)\left(1+\cos q_{z} d\right)
\end{aligned}
$$

where $d$ is the distance between the two $\mathrm{CuO}_{2}$ planes in $\mathrm{YBCO}$ compounds.

Note that for $\mathbf{q}=\mathbf{Q}_{\mathrm{AF}}=(\pi / a, \pi / a)$, which is the $\mathrm{AF}$ wavector of the undoped parent compounds of $\mathrm{YBa}_{2} \mathrm{Cu}_{3} \mathrm{O}_{6+x}$ and $\mathrm{La}_{2-x} \mathrm{Sr}_{x} \mathrm{Cu}_{2} \mathrm{O}_{4},{ }^{17} F_{\alpha}\left(\mathbf{Q}_{\mathrm{AF}}\right)$ and ${ }^{89} F_{\alpha}\left(\mathbf{Q}_{\mathrm{AF}}\right)$ are zero, whereas ${ }^{63} F_{\mathrm{c}}\left(\mathbf{Q}_{\mathrm{AF}}\right)$ and ${ }^{63} F_{a b}\left(\mathbf{Q}_{\mathrm{AF}}\right)$ present a maximum (Fig. 3). Let us suppose that AF fluctuations. 

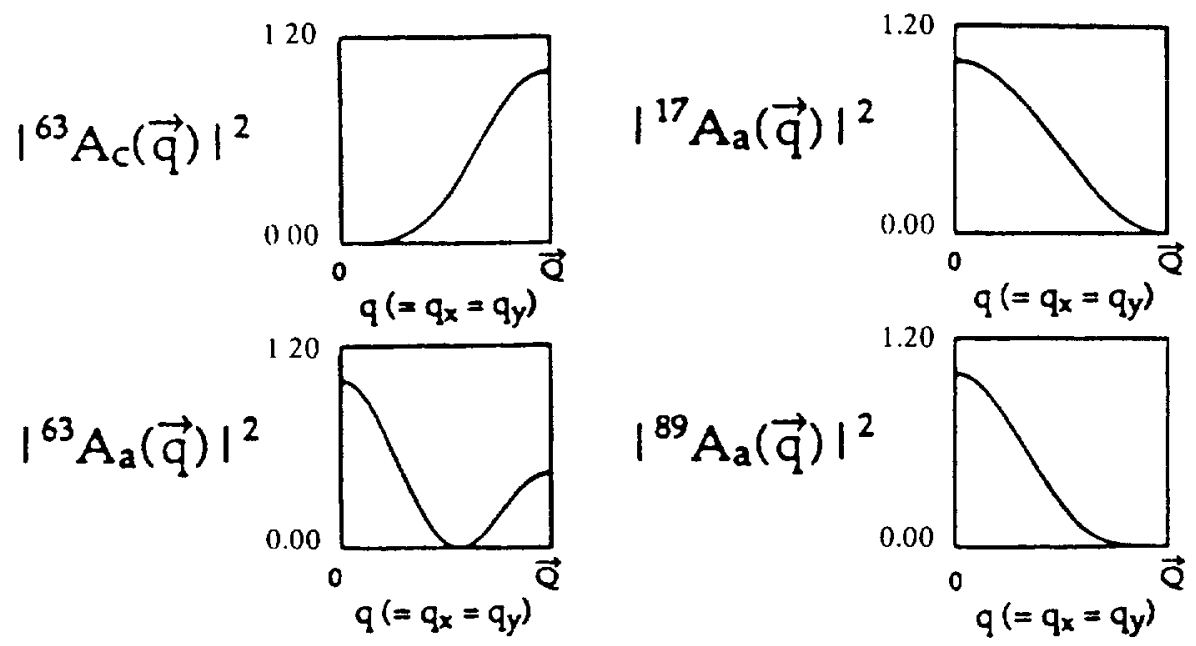

Fig. 3. - The $q$-dependence of the hyperfine form factors for planar ${ }^{63} \mathrm{Cu}$, planar ${ }^{17} \mathrm{O}$ and ${ }^{89} \mathrm{Y}$, derıved from the Shastry-Mila-Rice Hamiltonian. From Slichter et al. in reference [18].

reminiscent of the long range order present in the parent compounds, are present in the metallic phase, producing a peak around $\mathbf{Q}_{\mathrm{AF}}$ in the imaginary part of the dynamic spin susceptibility. Obviously, these AF fluctuations will strongly drive the copper NSLRR, but their contribution is heavily attenuated at the oxygen site, and still more so at the yttrium site. This purely geometrical effect, known for fifteen years in magnetic insulators [68], does not really depend on whether the copper spins are localized or not. On the contrary, the calculation of the anisotropy of the NSLRR using the form factors defined above in equation (10) fully assumes that the electronic spins can be considered as localized.

Another important consequence of the $q$-dependence of the Fourier transform of the hyperfine field is its effect on the nuclear spin-spin relaxation. By taking the Fourier transform of the expression given by equation (8), it turns out that the longitudinal component $A_{c}+2 B\left(\cos q_{x} a+\right.$ $\left.\cos q_{y} b\right)$ is much larger than the transverse one for $\mathbf{q}=\mathbf{Q}_{\mathrm{AF}}$. This, in addition to other arguments [49] justifies neglecting the transverse terms in the expression of $T_{2 \mathrm{G}}$ for $H_{0} / / c$ axis, which can then be written as $[69,70]$ :

$$
\left[\frac{1}{T_{2 \mathrm{G}}}\right]^{2}=\frac{c_{2}}{8 \hbar^{2}} \frac{\left(\gamma_{2} \hbar\right)^{4}}{\left(g \mu_{\mathrm{B}}\right)^{4}}\left[\sum_{q}\left|A_{c c}(q)\right|^{4} \chi^{2}(q)-\left\{\sum_{q}\left|A_{c c}(q)\right|^{2} \chi(q)\right\}^{2}\right]
$$

where $\imath$ stands for the copper 1sotope 63 or 65 and $c_{2}$ for their natural abundance. Because of the $q$-dependence of $A_{c c}$, which is maximum for $\mathbf{q}=\mathbf{Q}_{\mathrm{AF}}$ and close to zero for $q=0$, it is clear that in the case where $\chi(\mathbf{q})$ is peaked around $\mathbf{Q}_{\mathrm{AF}} . T_{2 \mathrm{G}}$ shows the temperature dependence of $\chi\left(\mathbf{Q}_{\mathrm{AF}}\right)$.

\section{Nuclear Spın-Lattice and Spın-Spın Relaxation in HTSC}

4.1. Nuclear Spin-Lattice Relaxation. - One of the most striking results obtained by NMR in HTSC, is the completely different temperature dependence observed for the copper and oxygen nuclei in the same $\mathrm{CuO}_{2}$ plane. This result is quite general. It is observed in all studied 


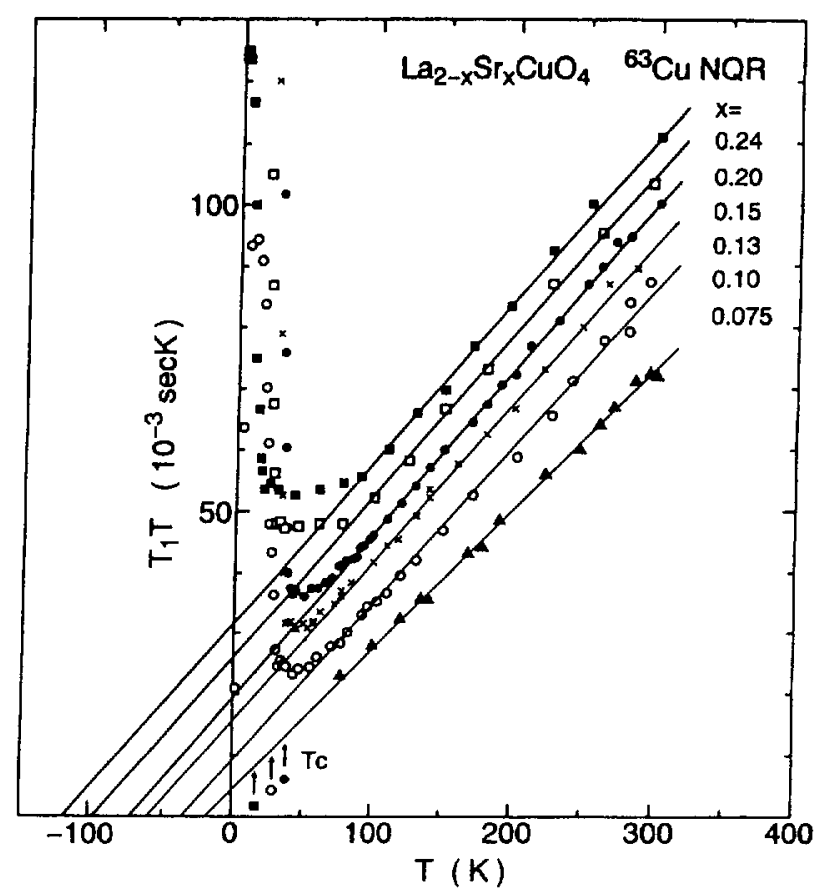

Fig. 4. $-{ }^{63} \mathrm{Cu} T_{1} T$ in $\mathrm{La}_{2-x} \mathrm{Sr}_{2} \mathrm{CuO}_{4}$ for various Sr contents. From Ohsugi et al. [71].

compounds, including overdoped compounds, although the more underdoped is the compound, the higher is the contrast. When starting from high temperature the Cu NSLRR divided by temperature, ${ }^{63}\left(T_{1} T\right)^{-1}$, increases when $T$ decreases. In the $\mathrm{La}_{2-x} \mathrm{Sr}_{\alpha} \mathrm{CuO}_{4}$ compounds, which can be studied on a larger temperature range than the other families. it has been clearly shown that ${ }^{63}\left(T_{1} T\right)^{-1}$ follows a Curie-Weiss behavior, or alternatively ${ }^{63}\left(T_{1} T\right)$ varies linearly with $T$ at high temperature [71] (Fig. 4). Still more striking are the results obtained by Imai et al. who have shown that above $\approx 700 \mathrm{~K} .1 /{ }^{63} T_{1}$ is constant and has the same value within $5 \%$, for $x$ ranging from 0 to 0.15 , that is from the pure antiferromagnetic parent compound to the optimally doped superconductor. This result seems to indicate that in the high temperature limit, the motion of the holes is not felt by the localized $\mathrm{Cu}$ spins. However, a slightly different result has been recently reported by the ISSP group [73], which claims that there is a $20 \%$ difference between the high temperature limit of $x=0$ and $x=0.15$, which they attribute to the motion of holes. In all other HTSC compounds, but for $\mathrm{YBa}_{2} \mathrm{Cu}_{4} \mathrm{O}_{8}$ [74], $\mathrm{Cu}$ NSLRR measurements have been performed at temperatures below $350 \mathrm{~K}$ and the Curie-Weiss behavior is not as well defined. For underdoped compounds, ${ }^{63}\left(T_{1} T\right)^{-1}$ passes through a maximum at a temperature $T^{*} \approx 150 \mathrm{~K}$ (for $\mathrm{YBCO}$ ) or higher (for $\mathrm{HgBa}_{2} \mathrm{Ca}_{2} \mathrm{Cu}_{3} \mathrm{O}_{8+\delta}$ [29]), that is well above $T_{\mathrm{c}}$, and then decreases with $T$, sometimes without a noticeable anomaly at $T=T_{\mathrm{c}}$. For overdoped compounds. ${ }^{63}\left(T_{1} T\right)^{-1}$ increases continuously as $T$ is decreased down to $T_{\mathrm{c}}$ (with some exceptions that we shall comment later), and then falls abruptly.

The situation is quite different for the NSLRR of the planar oxygen (Figs. 5 and 6 ). In the first approximation, ${ }^{17}\left(T_{1} T\right)^{-1}$ follows the same temperature dependence as the MHS (z.e.. the spin-susceptibility). It decreases with $T$ in the underdoped regime and is nearly constant at optimal doping. In the overdoped regime, the situation is not as clear, as in some cases it increases slightly with decreasing $T[14,75]$, whereas in others it decreases slightly [61]. 

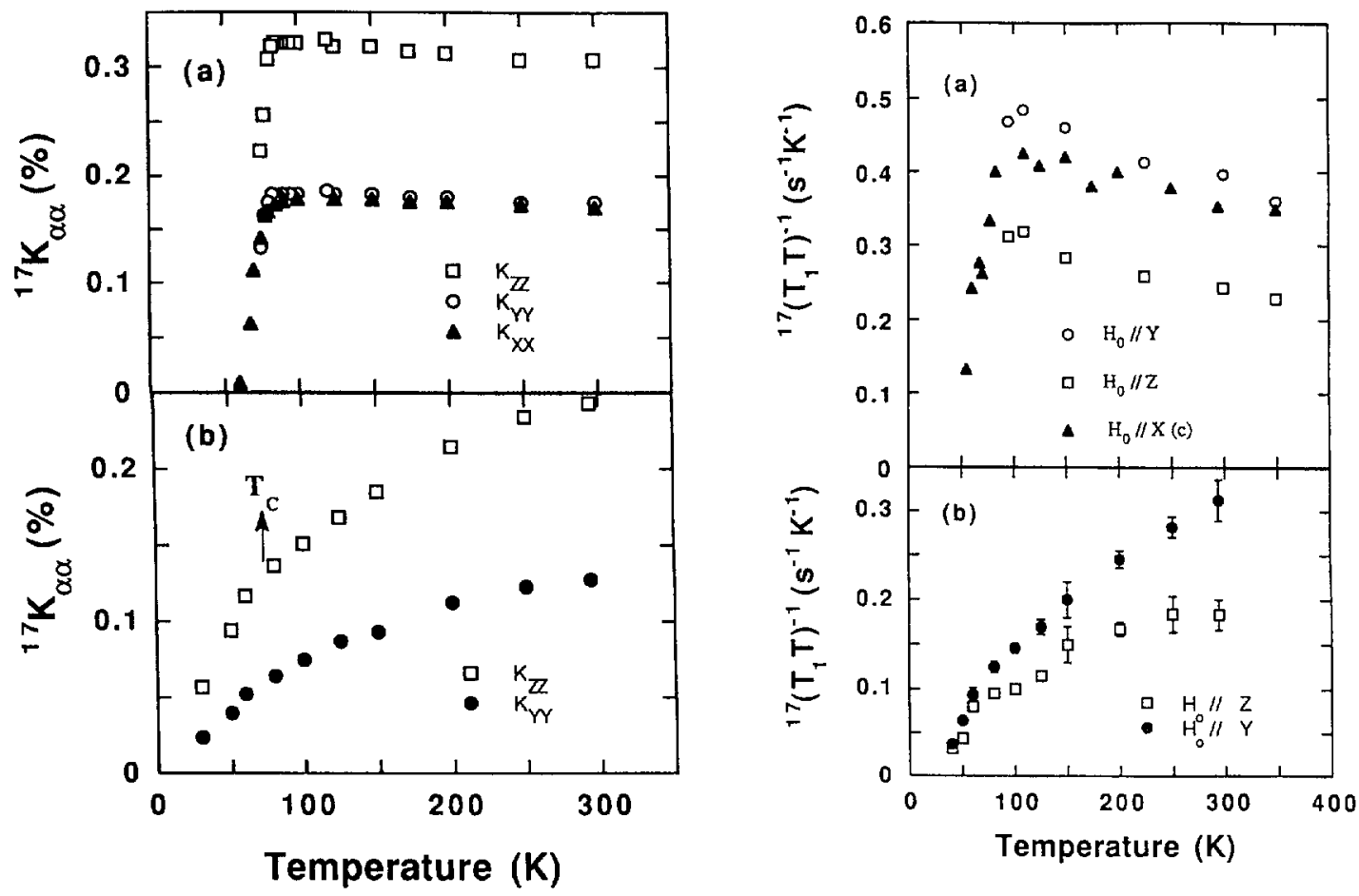

Fig. 5. - Left panel: magnetic hyperfine shift of planar ${ }^{17} \mathrm{O}$ for different field orientations in (a) $\mathrm{YBa}_{1}{ }_{92} \mathrm{Sr}_{0}{ }_{08} \mathrm{Cu}_{3} \mathrm{O}_{7}$ and (b) $\mathrm{YBa}_{2} \mathrm{Cu}_{3} \mathrm{O}_{6.52}$. Right panel: nuclear spin-lattice relaxation rate of planar ${ }^{17} \mathrm{O}$, for different field orientations in (a) $\mathrm{YBa}_{1}{ }_{92} \mathrm{Sr}_{0}{ }_{08} \mathrm{Cu}_{3} \mathrm{O}_{7}$ and (b) $\mathrm{YBa}_{2} \mathrm{Cu}_{3} \mathrm{O}_{6} 5_{2}$. From Horvatić et al. [46].

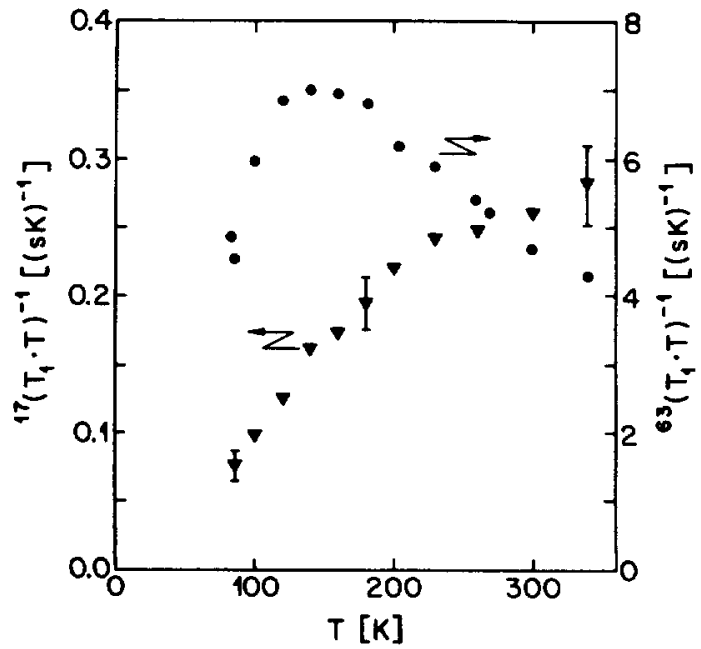

Fig. 6. - T-dependence of the planar ${ }^{63} \mathrm{Cu}$ and ${ }^{17} \mathrm{O}$ spin-lattice relaxation rates in $\mathrm{YBa}_{2} \mathrm{Cu}_{4} \mathrm{O}_{8}$. From Mangelschots et al. [55]. 


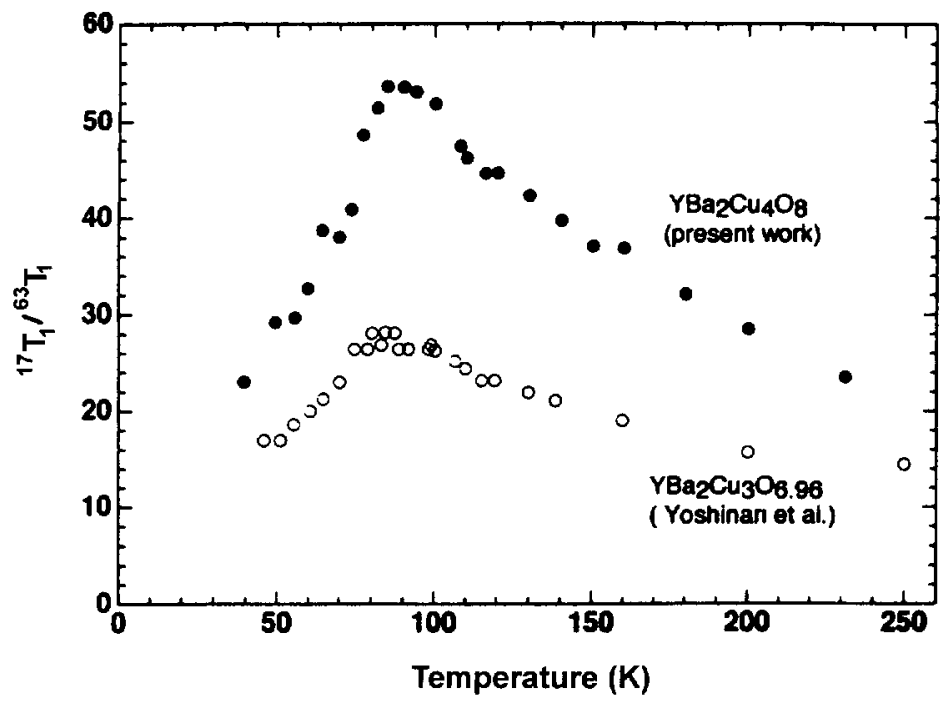

Fig. 7. - Temperature dependence of the ratio ${ }^{17} \mathrm{~T}_{1} /{ }^{63} \mathrm{~T}_{1}$. From Tomeno et al. [60].

In all cases, however, the ratio ${ }^{63}\left(T_{1} T\right)^{-1} /{ }^{17}\left(T_{1} T\right)^{-1}$ increases with decreasing $T$, with some more or less evident flattening near $T_{\mathrm{c}}$. Note that below $T_{\mathrm{c}}$, where the AF fluctuations disappear at low energy, the ratio ${ }^{63}\left(T_{1} T\right)^{-1} /{ }^{17}\left(T_{1} T\right)^{-1}$ falls down $[60,76]$ (Fig. 7). We shall see later that a similar temperature dependence is observed for ${ }^{63}\left(T_{2 \mathrm{G}}\right)^{-1}$, at least above $T_{\mathrm{c}}$. This sounds like the signature of the presence of AF fluctuations, even in overdoped compounds [61]. The sites between the $\mathrm{CuO}_{2}$ planes in bi-layers compounds, such as yttrium [52] in the YBCO family, or occasionally thallium as an impurity in $\mathrm{Tl}_{2} \mathrm{Ba}_{2} \mathrm{CaCu}_{2} \mathrm{O}_{8}$ [77], behave qualitatively and nearly quantitatively as the planar oxygen.

Because the Fermi liquid character of the normal state of HTSC cuprates has been strongly questioned, there has been an intense search for a Korringa relationship $K^{2} T_{1} T=$ const. for those nuclei which are less sensitive to AF fluctuations, like oxygen and yttrium. The quantitative comparison between the spin part of the MHS tensor and the NSLRR for oxygen or yttrium nuclei is not an easy task, snce it requires precise knowledge of the orbital MHS tensor. For most of the oxygen results obtained in the underdoped regime, plots of ${ }^{17}\left(T_{1} T\right)^{-1}$ or ${ }^{17}\left(T_{1} T\right)^{-1 / 2}$ versus ${ }^{17} K_{\alpha \alpha}$ exhibit a linear dependence within the error bars, simply leading to different values for ${ }^{17} K_{\text {orb }}$. In the first case, this leads to the relation $K T_{1} T=$ const., which can be interpreted as $\left(T_{1} T\right)^{-1}$ proportional to $\chi(T) / \Gamma$, where $\hbar / \Gamma$ is a temperature independent averaged correlation time. The other case leads to the more familiar Korrınga relationship, $K^{-2} T_{1} T=$ const. A similar situation is found for the yttrium data [52]. However. in spite of some recent interpretations in terms of Korringa behavior in the case of $\mathrm{YBa}_{2} \mathrm{Cu}_{4} \mathrm{O}_{8}[60,78]$, the validity of the relation $K T_{1} T=$ const. is commonly accepted, neglecting clear deviations at high temperature observed at the yttrium site $[65,79]$. Indeed, the ratio ${ }^{17}\left(T_{1} T\right) /^{89}\left(T_{1} T\right)$ has been found to increase by $30 \%$ between 300 and $100 \mathrm{~K}$ with decreasing temperature. in spite of the fact that ${ }^{17} K(T) /{ }^{89} K(T)$ is constant [65]. This unexpected temperature dependence is difficult to explain using the Mila-Rice Hamiltonian. At the same time, the anisotropy of NSLRR of the planar oxygen has been found $T$-dependent in the normal state of the " $60 \mathrm{~K}$ phase" of $\mathrm{YBa}_{2} \mathrm{Cu}_{3} \mathrm{O}_{6+x}[45,46]$, which is also impossible to explain within the framework of the Mila-Rice Hamiltonian [80]. 
Quite surprisingly, a magnetic field dependence of $T_{1}$ has been reported by Kambe et al. [81] in the single layer system $\mathrm{Tl}_{2} \mathrm{Ba}_{2} \mathrm{CuO}_{6+\delta}$, both below and above $T_{c}$. Soon after, part of their results has been confirmed by Vyaselev, Kolesnikov and Shegolev [82]. In fact, Kambe et al. have shown that $1 / T_{1} \propto 1 / \ln \left(H_{0}\right)$, a relation predicted for spin-diffusion processes in $2 \mathrm{D}$ paramagnets. Both groups have found that the more overdoped is the sample the larger is the effect. However, the fact that this behaviour is observed for ${ }^{63} \mathrm{Cu}$ and ${ }^{205} \mathrm{Tl}$ relaxation, which probe excitations around $\mathbf{Q}_{\mathrm{AF}}$, but not for ${ }^{17} \mathrm{O}$, which probes those around $q=0$, is particularly intriguing. Usual spin-diffusion processes are characterized by the low frequency divergence of $q \approx 0$ modes [83]. Here, something new seems to be combined, involving AF spin excitations, a fact by itself remarkable in such overdoped samples where the spin fluctuations are usually considered to be relatively weak and the system more three dimensional. It remains to be investigated whether such an effect can be produced by simple spin excitation diffusion or if it requires more sophisticated mechanisms, like for example, collective hole motion, including domain walls and antiphase spin domains [84]. In any case, we should learn quite a lot on the spin dynamics from this experiment, which has not yet received further experimental or theoretical clarification.

4.2. Nuclear Spin-Spin Relaxation Rate. - As already mentioned, the quantity $T_{2 \mathrm{G}}$ measures the strength of the indirect nuclear spin-spin interaction mediated by the non-local spin susceptibility. It has been measured on planar copper either by NQR or in high field experıments with $H_{0} / / c$-axis. It is a delicate measurement, particularly because to get reliable values of $T_{2 \mathrm{G}}$, it is necessary that the radiofrequency excitation covers the whole NMR or NQR line, which becomes technically difficult when the linewidth exceeds $200 \mathrm{kHz}$. Furthermore, the time dependence of the amplitude of the spin-echo is also strongly affected by an inelastic life-time contribution $1 / T_{1}^{*}$ so that $S(\tau)=\exp \left[-\tau^{2} /\left(2 T_{2 \mathrm{G}}^{2}\right)\right] \exp \left(-2 \tau / T_{1}^{*}\right)$. The $1 / T_{1}^{*}$, which is a linear combination of the NSLRR for $H_{0} / / c$-axis and for $H_{0} / / a b$-plane, has to be known accurately as a function of the temperature.

The temperature dependence of $1 / T_{2 \mathrm{G}}$ has been measured for various values of $x$ in $\mathrm{YBa}_{2} \mathrm{Cu}_{3}$ $\mathrm{O}_{6+r}[48,49,70,85,86]$, in $\mathrm{YBa}_{2} \mathrm{Cu}_{4} \mathrm{O}_{8}[48,87,88]$ and $\mathrm{Y}_{2} \mathrm{Ba}_{4} \mathrm{Cu}_{7} \mathrm{O}_{15}$ [87], in $\mathrm{Tl}_{2} \mathrm{Ba}_{2} \mathrm{CuO}_{6+\delta}$ [89]. $\mathrm{Tl}_{2} \mathrm{Ba}_{2} \mathrm{Ca}_{2} \mathrm{Cu}_{3} \mathrm{O}_{10}$ [62], $\mathrm{HgBa}_{2} \mathrm{Ca}_{2} \mathrm{Cu}_{3} \mathrm{O}_{8+\delta}$ [29.30] and $\mathrm{Bi}_{2} \mathrm{Sr}_{2} \mathrm{CaCu}_{2} \mathrm{O}_{8}$ [64]. It has also been measured in the paramagnetic phase of $\mathrm{La}_{2} \mathrm{CuO}_{4}$ [90], where it has been found proportional to ${ }^{63}\left(T_{1} T\right)^{-1}$ and quite recently in $\mathrm{La}_{2-2} \mathrm{Sr}_{x} \mathrm{CuO}_{4}[91]$. In all cases, 1 t has been found to increase with decreasing temperature, with sometimes a flattening or a small decrease close to $T_{\mathrm{c}}$. This can be safely considered as a proof for the existence of AF correlations in all these compounds. As we shall see later, the comparison of the temperature dependence of $1 / T_{2 \mathrm{G}}$ and ${ }^{63}\left(T_{1} T\right)^{-1}$ is essential in the interpretation of the NMR data (Fig. 8).

Finally, we would like to mention a creative experiment performed in $\mathrm{Y}_{2} \mathrm{Ba}_{4} \mathrm{Cu}_{7} \mathrm{O}_{15}$, a compound which can be vewed as a natural superlattice consisting of alternating blocks of $\mathrm{YBa}_{2} \mathrm{Cu}_{4} \mathrm{O}_{8}$ and $\mathrm{YBa}_{2} \mathrm{Cu}_{3} \mathrm{O}_{i}[87,90]$. This situation makes the two $\mathrm{CuO}_{2}$ planes of a bilayer structurally inequivalent. Thus, one obtains two different $\mathrm{Cu}$ NMR resonance lines, each corresponding to one plane of the bi-layer. It offers the unique opportunity to perform a spın-echo double resonance (SEDOR) experiment: while performing a classical spin-echo pulse sequence $(\pi / 2, \pi)$ on one of the two $\mathrm{Cu}$ lines, one also applies a $(\pi)$ pulse at the resonance frequency of the other line, i.e., the nuclear spins are flipped in the second plane. By observing a reduction of the size of the spin-echo when applying the second pulse, Stern et al. [93] have shown that nuclear spins in one plane could be depolarized by electronic spins in the other plane [94]. In other words, there exists an electronic coupling $J_{\perp}$ between the two planes. From this experiment. Millıs and Monien have deduced that $J_{\perp}=14 \mathrm{meV}$ [95], in good agreement 

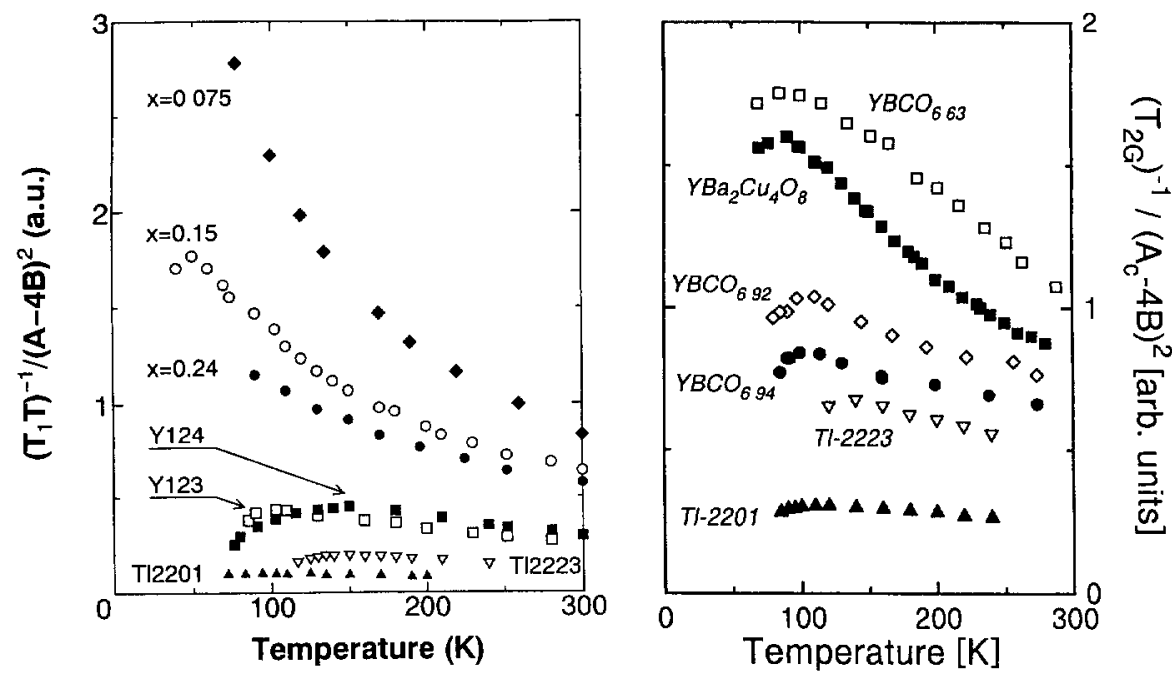

Fig. 8. - Left panel: temperature dependence of $\left(T_{1} T\right)^{-1} /(A-4 B)^{2}$ in various cuprates (courtesy of Kitaoka). Right panel: the $T$-dependence of $\left(T_{2 G}\right)^{-1} /(A-4 B)^{2}$ in $\mathrm{YBa}_{2} \mathrm{Cu}_{3} \mathrm{O}_{6} 63$ [70], $\mathrm{YBa}_{2} \mathrm{Cu}_{4} \mathrm{O}_{8}$ [48], $\mathrm{YBa}_{2} \mathrm{Cu}_{3} \mathrm{O}_{6.92}$ [85], $\mathrm{YBa}_{2} \mathrm{Cu}_{3} \mathrm{O}_{6} 94$ [86], slightly underdoped $\mathrm{Tl}_{2} \mathrm{Ba}_{2} \mathrm{Ca}_{2} \mathrm{Cu}_{3} \mathrm{O}_{10}$ [62], and overdoped $\mathrm{Tl}_{2} \mathrm{Ba}_{2} \mathrm{CuO}_{6+\delta}[89]$.

with the recent value of $10 \mathrm{meV}$ inferred from the measurement of the optical magnon dispersion in $\mathrm{YBa}_{2} \mathrm{Cu}_{3} \mathrm{O}_{6.2}[96]$.

4.3. Quantitative Analysis of the Nuclear Relaxation. - The striking difference in the temperature dependence of the NSLRR for the copper and oxygen sites in the $\mathrm{CuO}_{2}$ planes of $\mathrm{YBa}_{2} \mathrm{Cu}_{3} \mathrm{O}_{7}$ [40] was immediately identified as a proof for the existence of $\mathrm{AF}$ fluctuations in the whole metallic range of the $\mathrm{YBa}_{2} \mathrm{Cu}_{3} \mathrm{O}_{6+x}$ and a consequence of the form factor of the oxygen. However, to go further in the interpretation required a model for the dynamic spin susceptibility. There have been many theoretical works devoted to the NSLR in the cuprates [97]. In this section, we shall only consider two of them: 1) the nearly antiferromagnetic Ferm1 liquid model (NAFL), proposed independently by Millis Monien and Pines (MMP) [98.99] and Moriya, Takahashi and Ueda [100]. i1) the model based on the scaling properties of a disordered 2D Heisenberg antiferromagnet [101,102]. These models allow a quantitative analysis of the NSLR and NSSR data, and thus a comparison between the various compounds. For other types of model, the reader is referred to references $[84,97,103]$; some of them w1ll be discussed in Section 6.

MMP have proposed a phenomenological expression within the RPA approxımation:

$$
\chi^{\prime \prime}\left(Q_{\mathrm{AF}}+q, \omega_{\mathrm{n}}\right)=\frac{\pi \omega_{\mathrm{n}} \chi_{0}(T)}{\Gamma_{0}}+\frac{\omega_{\mathrm{n}} \beta \chi_{0}\left(Q_{\mathrm{AF}}\right) \xi^{4}}{\Gamma_{0}\left(Q_{\mathrm{AF}}\right)\left(1+\xi^{2} q^{2}\right)^{2}}=\chi_{\mathrm{QP}}^{\prime \prime}+\chi_{\mathrm{AF}}^{\prime \prime},
$$

where $\chi_{\mathrm{AF}}^{\prime \prime}$ describes the contribution of the AF fluctuations, $\chi_{\mathrm{QP}}^{\prime \prime}$ is a background contribution of the quasi-particles. $\chi_{0}(T)$ is the macroscopic susceptibility, $\hbar \Gamma_{0}$ and $\hbar \Gamma_{0}\left(\mathbf{Q}_{\mathrm{AF}}\right)$ are characteristic energies of the unperturbed Fermı liqu1d, $\beta$ represents the strength of the $A F$ fluctuations, and $\xi$ is the static correlation length. The susceptibility $\chi_{\mathrm{AF}}^{\prime \prime}$ can be rewritten as:

$$
\chi_{\mathrm{AF}}^{\prime \prime}\left(Q_{\mathrm{AF}}+q, \omega_{\mathrm{n}}\right)=\frac{\omega_{\mathrm{n}} \chi_{\mathrm{AF}}\left(Q_{\mathrm{AF}}+q\right)}{\Gamma\left(Q_{\mathrm{AF}}+q\right)}
$$


with

and

$$
\chi_{\mathrm{AF}}\left(Q_{\mathrm{AF}}+q\right)=\frac{\beta^{1 / 2} \chi_{0}\left(Q_{\mathrm{AF}}\right) \xi^{2}}{\left(1+\xi^{2} q^{2}\right)}=\frac{\alpha \xi^{2}}{\left(1+\xi^{2} q^{2}\right)}
$$

$$
\Gamma\left(Q_{\mathrm{AF}}\right)=\Gamma_{0}\left(Q_{\mathrm{AF}}\left(1+\xi^{2} q^{2}\right) / \beta^{1 / 2} \xi^{2}\right.
$$

For large values of $\xi / a(\xi / a \geq 2),{ }^{63}\left(T_{1} T\right)^{-1}$ in this model varies as $(\xi / a)^{2}$, which is made to vary as $1 / T$ in order to fit the NMR data. In addition, large values of $\xi / a$ ensure an efficient filterıng of the contribution of the AF fluctuations at the oxygen and yttrium sites, the relaxation of these nuclei being thus governed by $\chi_{\mathrm{QP}}^{\prime \prime}$. This model obviously fits the data [104] for a $\Gamma_{0}$ value approximately constant since for these nuclel. $\left(T_{1} T\right)^{-1}$ is roughly proportional to $\chi_{0}(T)$.

By extending their self-consistent renormalization theory of spin-fluctuations to 2-D, Moriya, Takahashi and Ueda [100] have derived independently a rather similar model. Their calculations provides values of $(\xi / a)^{2}$ that vary as $1 / T$. The correspondence between the parameters in these two theories of nearly antiferromagnetic Fermi liquid are:

$$
\beta=\frac{q_{\mathrm{B}}^{4}}{4 \pi \alpha_{\mathrm{S}} T_{\mathrm{A}} T_{0}}, \quad \Gamma\left(Q_{\mathrm{AF}}\right)=2 \pi T_{0} \xi^{-2} q_{\mathrm{B}}^{-2}, \quad \chi\left(Q_{\mathrm{AF}}\right)=\frac{q_{\mathrm{B}}^{2} \xi^{2}}{2 \alpha_{\mathrm{S}} T_{\mathrm{A}}}
$$

where $q_{\mathrm{B}}$ is a cutoff wave vector of the order of $\pi / a, \alpha_{\mathrm{S}}$ is related to the enhancement of the staggered susceptibility, and $T_{\mathrm{A}}$ and $T_{0}$ are two characteristic energies $\left(\hbar=k_{\mathrm{B}}=1\right)$.

A quite different model, based upon scaling analysis of the phase diagram of a disordered $2 \mathrm{D}$ Heisenberg antiferromagnet has been proposed by Sokol and Pines [101]. Following Chubukov and Sachdev [102], Pines and collaborators [105] assume that below some temperature corresponding to a correlation length value $\xi / a \approx 2$, the cuprates in the underdoped regime belong to the quantum critical regime defined by Chakravarty, Halperin and Nelson [106]. Within this regime, both the static susceptibility and $\xi / a$ vary as $T^{-1}$ and the only characteristic energy is set by the temperature. Below a particular temperature, the system enters into the quantum disordered regime; that is, $\xi / a$ becomes constant and a spin gap opens with a value $\Delta \approx c / \xi$, where $c$ is related to the spin wave velocity. In this description, the parameter $g$, which is a measure of the strength of the disorder, is larger than the critical value $g_{\mathrm{c}}$ which separates the long range ordered from the quantum disordered ground states at $T=0$. The parameter $g$ is assumed to increase with the hole concentration $n_{\mathrm{h}}$ in the plane [10 $\tau$ ]. When $n_{\mathrm{h}}$ becomes too large, Sokol and Pines have suggested that magnon-type spin excitations present in the system become overdamped, and that the NAFL regime should be recovered.

By using scaling arguments, one may assume, following Sokol and Pines, that $\chi\left(Q_{\mathrm{AF}}+q\right)=$ $\chi\left(Q_{\mathrm{AF}}\right) f(\xi q)$ with $\chi\left(Q_{\mathrm{AF}}\right)=\alpha \xi^{2-\eta}$ with $\eta \approx 0$ and $\alpha$ a constant [108]. This relation imples from equation (11) that $1 / T_{2 \mathrm{G}}$ is proportional to $\xi^{1-\eta}$ in both descriptions. On the other hand,

$$
\lim _{\omega \rightarrow 0} \operatorname{Im} \chi(q, \omega) / \omega=\frac{\chi\left(Q_{\mathrm{AF}}+q\right)}{\Gamma\left(Q_{\mathrm{AF}}+q\right)} \propto \frac{\alpha \xi^{2-\eta} f(\xi q)}{\xi^{-z} \Gamma_{\mathrm{AF}} g(\xi q)},
$$

where $z$ is the dynamic critical exponent which describes the variation of the characteristic energy as a function of $\xi$, z.e., $\Gamma\left(Q_{\mathrm{AF}}+q\right)=\Gamma_{\mathrm{AF}} \xi^{-z} g(\xi q)$. After summation over the Brillouin zone, ${ }^{63}\left(T_{1} T\right)^{-1}$ is proportional to $\alpha \xi^{z-\eta} / \Gamma_{\mathrm{AF}}$.

In the quantum critical regime $z=1$, so that $\frac{T_{1} T}{T_{2 \mathrm{G}}}$ must be independent of the temperature and proportional to $\Gamma_{\mathrm{AF}}[109]$. In the case of the NAFL, we see easily from equations (13, 14) that $z=2$ (and $\eta=0$ ), so that it is now $\frac{T_{1} T}{\left(T_{2 G}\right)^{2}}$ which must be temperature independent 


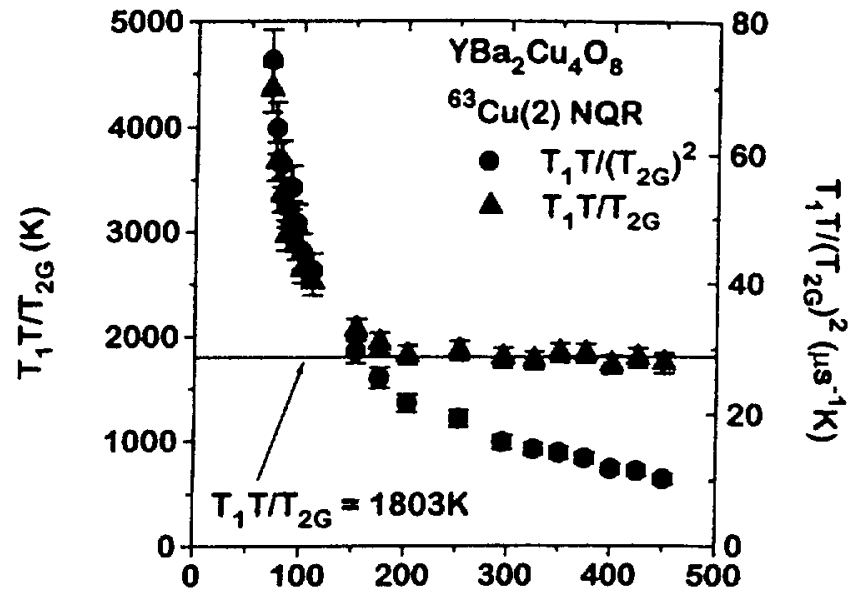

Fig. 9. - Ratios $T_{1} T / T_{2 \mathrm{G}}$ and $T_{1} T /\left(T_{2 \mathrm{G}}\right)^{2}$ for ${ }^{63} \mathrm{Cu}$ in $\mathrm{YBa}_{2} \mathrm{Cu}_{4} \mathrm{O}_{8}$. From Corey et al. [88].

and proportional to $\Gamma_{\mathrm{AF}} \chi\left(Q_{\mathrm{AF}}\right)$, the prefactor depending only on the hyperfine couplings. Experimentally, in the metallic part of the phase diagram, $T_{1} T / T_{2 \mathrm{G}}=$ const. seems to be obeyed for all underdoped compounds, for temperatures above the spin gap regime, if one takes the raw experimental value of ${ }^{63} T_{1} T$. A very good example is the case of $\mathrm{YBa}_{2} \mathrm{Cu}_{4} \mathrm{O}_{8}[48,87,88]$ for which the results of reference [88] are shown in Figure 9. However, if one subtracts from the experimental value the contribution of $\chi_{\mathrm{QP}}=\frac{\omega_{\mathrm{n}} \pi \chi_{0}(T)}{\Gamma_{0}}$, (this can be done by multiplying the planar oxygen NSLRR ${ }^{17}\left(T_{1} T\right)^{-1}$ by an appropriate coefficient $k$ which can be directly derived from the hyperfine coupling constant so that ${ }^{63}\left(T_{1} T\right)_{\mathrm{AF}}^{-1}={ }^{63}\left(T_{1} T\right)_{\exp }^{-1}-\mathrm{k}^{17}\left(T_{1} T\right)_{\exp }^{-1}$, it seems that the relation $T_{1} T /\left(T_{2 \mathrm{G}}\right)^{2}=$ const. is well obeyed for temperatures above the spin-gap regime, as expected in the NAFL approach. This latter type of interpretation has been promoted and systematically adopted by the ISSP group [110]. This approach. however, depends on the valıdity of the single spin fluid model to explain the spin dynamics, a question which is far from being obvious.

The great majority of the nuclear relaxation data has been quantitatively analyzed within the framework of these two models, which permit to evaluate the values of $\chi\left(\mathbf{Q}_{\mathrm{AF}}\right)$ and $\Gamma\left(\mathbf{Q}_{\mathrm{AF}}\right)$. from those of $T_{1} T$ and $T_{2 \mathrm{G}}$. Both models suppose that $\xi$ is temperature dependent, which is not obvious as we shall see later. Whatever is the chosen procedure. there are strong indications that the value of the characteristic energy $\Gamma\left(\mathbf{Q}_{\mathrm{AF}}\right)$ correlates with the optimal value of $T_{\mathrm{c}}$ in a given system [29.30,62].

\section{Spin Gap}

As already emphazised in the introduction, the evidence for a pseudogap in the spin excitations of underdoped compounds has been a major outcome of NMR investigations in the normal state of the cuprates. A large amount of data have been accumulated, and in the past years, evidence has been presented for a pseudo gap showing up in many physical properties, involving both spin and charge degrees of freedom. Consequently, the striking difference between the underdoped and overdoped regimes is increasingly debated. and is now considered as one of the very central questions in the physics of the cuprates [111]. Since the early days, the notion 
of spin (pseudo) gap has considerably evolved and contradictory definitions can be found in the literature. Therefore, we first survey the experimental situation from the NMR point of view, and discuss some connections with other experimental techniques.

Originally, the term "spin-gap" referred to the peculiar $T$-dependence of the quantity ${ }^{63}\left(T_{1} T\right)^{-1}$ which is proportional to the dynamic susceptibility $\chi "\left(\mathbf{Q}_{\mathrm{AF}}, \omega_{\mathrm{n}}\right)$. Indeed, it was early discovered in underdoped $\mathrm{YBa}_{2} \mathrm{Cu}_{3} \mathrm{O}_{6+x}$, that ${ }^{63}\left(T_{1} T\right)^{-1}$ passes through a maximum around $130-150 \mathrm{~K}$ that is well above $T_{\mathrm{c}}$, and then decreases with temperature $[112,113]$. This feature was first identified as a loss in the spectral weight of the low energy antiferromagnetic excitations by Yasuoka et al. [114]. Later on, the opening of such a gap for the spin excitations at $\mathbf{Q}_{\mathrm{AF}}=(\pi / a, \pi / a)$ was confirmed by inelastic neutron scatterıng [115], and finally recognized as a characteristic feature of underdoped compounds [14,116]. although the situation was not clear in the $\mathrm{La}_{2-x} \mathrm{Sr}_{x} \mathrm{CuO}_{4}$ system [71]. Here, we caution the reader that the expression "spingap" is sometimes used in the neutron scattering community to describe the low temperature behaviour of $\chi$ " $\left(\mathbf{Q}_{\mathrm{AF}}, \omega\right)$ far below $T_{\mathrm{c}}(4.2 \mathrm{~K}$ in general), where a true gap is indeed observed presumably related to the superconducting one. However, this definition cannot account for the features observed above $T_{\mathrm{c}}$ by NMR, which actually measures:

$$
\frac{\chi^{\prime \prime}\left(\mathbf{Q}_{\mathrm{AF}}, \omega_{\mathrm{n}}\right)}{\omega_{\mathrm{n}}}=\lim _{\omega \rightarrow 0}\left(\frac{\partial \chi^{\prime \prime}\left(\mathbf{Q}_{\mathrm{AF}}, \omega\right)}{\partial \omega}\right),
$$

ı.e., the slope of $\chi^{\prime \prime}\left(\mathbf{Q}_{\mathrm{AF}, \omega}\right)$ at $\omega=0$. In fact, there is no discrepancy at all between $\mathrm{Cu}$ NMR and neutron results, since with decreasing $T$, a gradual decrease of this slope is indeed observed by inelastic neutron scattering (INS) in the normal state of $\mathrm{YBa}_{2} \mathrm{Cu}_{3} \mathrm{O}_{6+x}$ [115]. This effect is then referred to as a "pseudogap" since what is really observed in INS experiments is a progressive transfer of the spectral weight from low to high energies. This spin pseudogap appears to be the only quantity one should compare to ${ }^{63}\left(T_{1} T\right)^{-1}$. In NMR experiments, the above described removal of spectral weight has been later confirmed through measurements of the indirect spin-spin relaxation rate $T_{2 \mathrm{G}}$, which reflects the temperature dependence of the staggered susceptibility at $\mathbf{Q}_{\mathrm{AF}}$ [48]. This result is easy to understand if one considers $[48,85]$. that ${ }^{63}\left(T_{1} T\right)^{-1}$ is proportional to $\chi "\left(\mathbf{Q}_{\mathrm{AF}}, \omega\right) / \omega$ for $\omega \approx 0$ whereas $\left(T_{2 \mathrm{G}}\right)^{-1}$ is proportional to $\chi^{\prime}\left(\mathbf{Q}_{\mathrm{AF}}, 0\right)$, i.e., to $\int_{-\infty}^{+\infty} \frac{\chi^{\prime \prime}\left(\mathbf{Q}_{\mathrm{AF}}, \omega\right)}{\omega} \mathrm{d} \omega$. As long as the frequency dependence of $\chi "\left(\mathbf{Q}_{\mathrm{AF}}, \omega\right) / \omega$ is not modified, the two quantities are proportional. As soon as a pseudogap opens, however, the amplitude of $\chi "\left(\mathbf{Q}_{\mathrm{AF}}, \omega\right) / \omega$ at the origin will decrease much faster than the integral, so the ratio ${ }^{63}\left(T_{1} T\right){ }^{63}\left(T_{2 \mathrm{G}}\right)$ will increase (Fig. 10). It is important to realize that this ratio will always show a rapid varlation at the opening of the gap, whatever is the actual form of $\chi^{\prime \prime}\left(\mathbf{Q}_{\mathrm{AF}}, \omega\right)$. Experimentally, this modification of the ratio in ${ }^{63}\left(T_{1} T\right) /{ }^{63}\left(T_{2 \mathrm{G}}\right)$ has been clearly observed in all underdoped compounds for which it has been measured: $\mathrm{YBa}_{2} \mathrm{Cu}_{4} \mathrm{O}_{8}$ $[48,87,88], \mathrm{YBa}_{2} \mathrm{Cu}_{3} \mathrm{O}_{6.63}$ [70], $\mathrm{Y}_{2} \mathrm{Ba}_{4} \mathrm{Cu}_{7} \mathrm{O}_{15}$ [87], $\mathrm{YBa}_{2} \mathrm{Cu}_{3} \mathrm{O}_{6} 92$ [85], $\mathrm{Tl}_{2} \mathrm{Ba}_{2} \mathrm{Ca}_{2} \mathrm{Cu}_{3} \mathrm{O}_{10}$ [62], $\mathrm{HgBa}_{2} \mathrm{Ca}_{2} \mathrm{Cu}_{3} \mathrm{O}_{8+\delta}$ [29]. In contrast, this ratio has been shown to be constant or smoothly varying, with a sharp modification occurring only at the superconducting transition, for the optimally doped $\mathrm{YBa}_{2} \mathrm{Cu}_{3} \mathrm{O}_{694}\left(T_{\mathrm{c}}=93 \mathrm{~K}\right)$ [86], $\mathrm{HgBa}_{2} \mathrm{Ca}_{2} \mathrm{Cu}_{3} \mathrm{O}_{8+\delta}$ and for the overdoped compounds $\mathrm{YBa}_{2} \mathrm{Cu}_{3} \mathrm{O}_{6} 98$ [48,117], $\mathrm{Bi}_{2} \mathrm{Sr}_{2} \mathrm{CaCu}_{2} \mathrm{O}_{8}$ [64] and $\mathrm{Tl}_{2} \mathrm{Ba}_{2} \mathrm{CuO}_{6+\delta}$ [89]. The case of these two latter compound deserves to be emphasized, since ${ }^{63}\left(T_{1} T\right)^{-1}$ exhibits a maximum above $T_{\mathrm{c}}$, but the ratio ${ }^{63}\left(T_{1} T\right) /{ }^{63}\left(T_{2 \mathrm{G}}\right)$ is constant. Consequently, the decrease of ${ }^{63}\left(T_{1} T\right)^{-1}$ is currently believed to have another orıgin, that is not yet clarified (the uniform spin susceptibility also shows a maximum around $120 \mathrm{~K}$ ). Experımentally it therefore appears, at least for bi-layer and tri-layer compounds, that the opening of a spin-gap at $\mathbf{q}=\mathbf{Q}_{\mathrm{AF}}$ is only present in underdoped compounds and that the optimal composition is a singular point in the phase diagram as a function of the hole concentration. In order to draw more quantitative information 


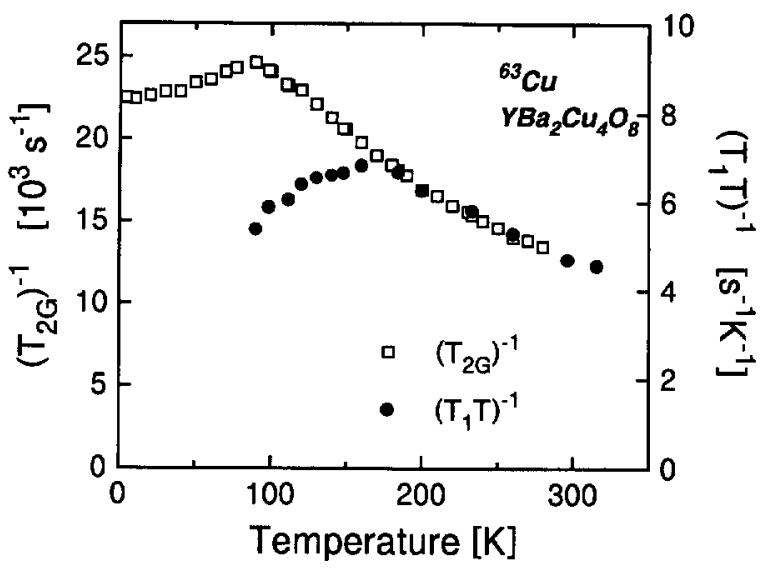

Fig. 10. $-\left(T_{1} T\right)^{-1}$ and $\left(T_{2 G}\right)^{-1}$ from ${ }^{63} \mathrm{Cu} \mathrm{NQR} \mathrm{in} \mathrm{YBa}_{2} \mathrm{Cu}_{4} \mathrm{O}_{8}$. Data from Itoh et al. [48].

from the NMR data, such as the doping dependence of the cross-over temperature $T^{*}$ corresponding to the opening of this spin-gap, one has to choose a criterion to define $T^{*}$. Several definitions have been proposed: one is the temperature at which ${ }^{63}\left(T_{1} T\right)^{-1}$ is maximum, the other is the deviation of ${ }^{63}\left(T_{1} T\right)$ from its linear $T$-dependence. The case of the optimally doped compound [85] shows that these two definitions may be hazardous close to the optimal concentration [118], a safer definition being to define $T^{*}$ from the temperature dependence of the ratio ${ }^{63} T_{1} T /^{63} T_{2 \mathrm{G}}$. From the maximum of ${ }^{63}\left(T_{1} T\right)^{-1}$, Machi et al. [119] in $\mathrm{Y}_{1-x} \mathrm{Ca}_{x} \mathrm{Ba}_{2} \mathrm{Cu}_{4} \mathrm{O}_{8}$ and Matsumura et al. [120] in $\mathrm{Y}\left(\mathrm{Ba}_{1-x} \mathrm{La}_{x}\right){ }_{2} \mathrm{Cu}_{3} \mathrm{O}_{y}$ found a negative linear dependence of $T^{*}$ on the hole concentration, which roughly extrapolates to $T_{\mathrm{c}}$ for the optimal concentration. On the other hand, Berthier and collaborators have found that $T^{*}=T_{\mathrm{c}}$ in optimally doped $\mathrm{YBa}_{2} \mathrm{Cu}_{3} \mathrm{O}_{6} 94\left(T_{\mathrm{c}}=93 \mathrm{~K}\right)$ [121], and that $T^{*}=150 \mathrm{~K}$ in a slightly underdoped sample having $T_{\mathrm{c}}$ as high as $91 \mathrm{~K}$ [85] (Fig. 11). These observations suggest a rapid variation of $T^{*}$ close to the optimal value of $T_{\mathrm{c}}$. Altogether, one must recognize that rather little is known concerning the (presumably weak) dependence of $T^{*}$ on the carrier concentration. Recently, a surprisingly high $T^{*}(\cong 230 \mathrm{~K})$ has been found in underdoped $\mathrm{HgBa}_{2} \mathrm{Ca}_{2} \mathrm{Cu}_{3} \mathrm{O}_{8+\delta}$ with $T_{\mathrm{c}}=115 \mathrm{~K}$ [29] (the optimal $T_{\mathrm{c}}$ value at ambient pressure is $135 \mathrm{~K}$ ). This finding, along with the fact that high values for $\Gamma_{\mathrm{AF}}$ and the super-transferred hyperfine field $B$ have also been reported in the same compound [29.30]. strongly suggest that the AF spin fluctuations play an important role in the pairıng mechanism.

Let us now turn to the static uniform spin susceptibility $\chi(q=0, \omega=0)=\chi_{0}$, as probed by the magnetic hyperfine shift of varıous nuclei. This information is basically the same as that obtained by measuring the macroscopic susceptibility, but it is often more accurate since NMR is not sensitive to additional macroscopic spurrous phases. In many compounds the quantity $\chi_{0}$ exhibits an anomalous decrease with $T$ below some temperature $T_{0}$, well above $T_{\mathrm{c}}$ (Fig. 12). Actually, the decrease at high temperature is not sharp, and looks rather like the opening of a pseudogap. However, the slope becomes steeper and steeper with decreasing $T$ and there is only small change of the curvature in the vicinity of $T_{\mathrm{c}}$. It is possible, but not proved, that the more rapid fall off starts at $T^{*}[105,122]$. These features are quite unversal for underdoped compounds, whatever is the number of $\mathrm{CuO}_{2}$ planes per unit cell, but they are also sometimes observed in slightly overdoped compounds, like $\mathrm{Tl}_{2} \mathrm{Ba}_{2} \mathrm{CuO}_{6+\delta}$ $\left(T_{\mathrm{c}}=85 \mathrm{~K}\right)$ [61] or $\mathrm{Bi}_{2} \mathrm{Sr}_{2} \mathrm{CaCu}_{2} \mathrm{O}_{8}$ [62]. Also, great care must be taken for compounds close 


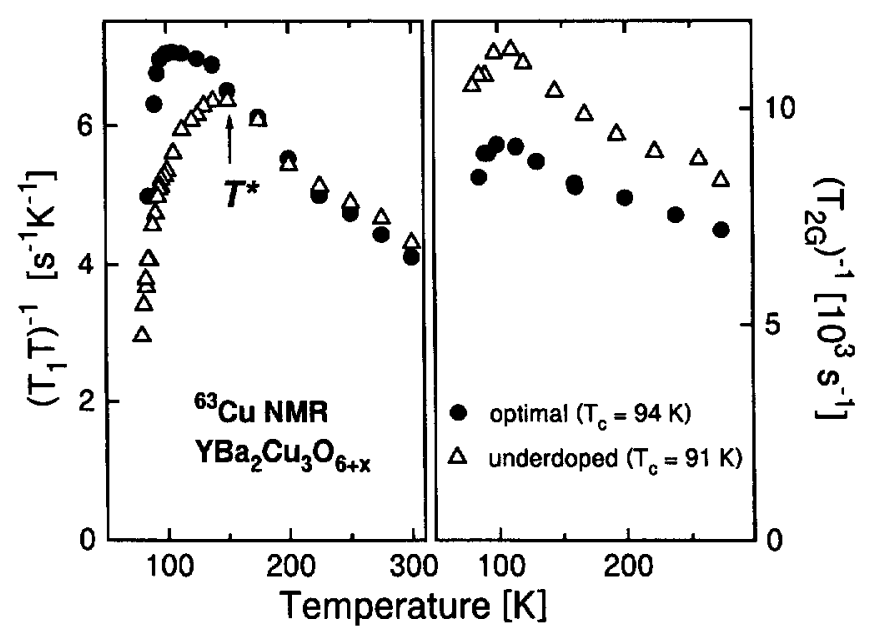

Fig. 11. $-\left(T_{1} T\right)^{-1}$ and $\left(T_{2} G\right)^{-1}$ measured in the slightly underdoped $\mathrm{YBa}_{1}{ }_{93} \mathrm{Sr}_{0}{ }_{07} \mathrm{Cu}_{3} \mathrm{O}_{6} 92$ with $T_{\mathrm{c}}=91 \mathrm{~K}$ (triangles) and in the optimally doped $\mathrm{YBa}_{2} \mathrm{Cu}_{3} \mathrm{O}_{6} 94$ with $T_{\mathrm{c}}=93 \mathrm{~K}$ (circles). Data from Gillet et al. [121].

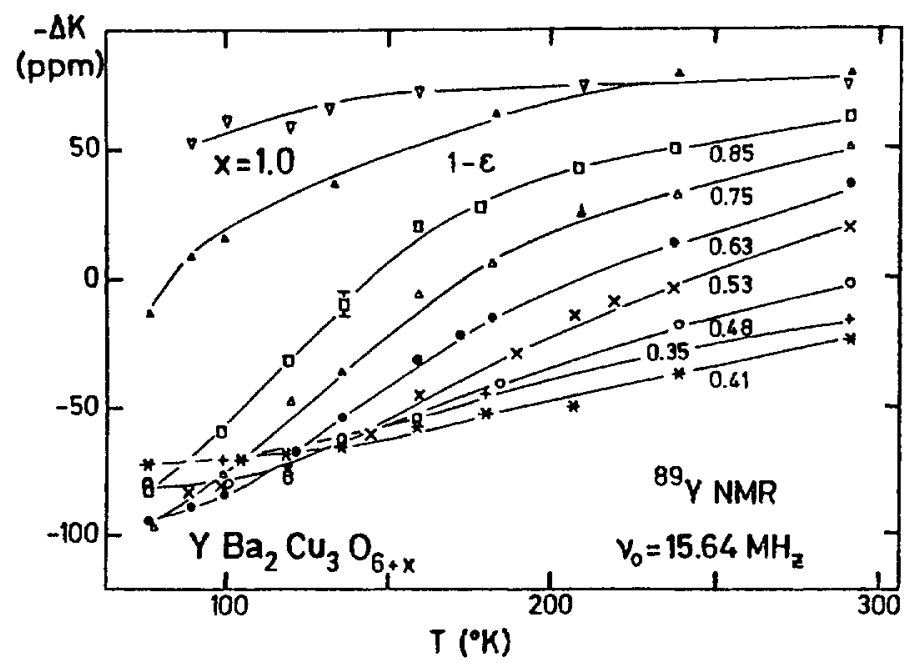

Fig. 12. $-{ }^{89} \mathrm{Y}$ magnetic hyperfine shift in $\mathrm{YBa}_{2} \mathrm{Cu}_{3} \mathrm{O}_{6+x}$ for various oxygen contents. From Alloul et al. [52].

to or at the optimal doping, where a decrease above $T_{c}$ might be related to a precursor effect of the superconducting transition (For example, in overdoped $\mathrm{YBa}_{2} \mathrm{Cu}_{3} \mathrm{O}_{7}[65,79]$ a maximum is observed close to $T_{\mathrm{c}}$ in the yttrium MHS). The values of $T_{0}$ usually range from $T_{\mathrm{c}}$ for samples near optimal doping, to more than $400 \mathrm{~K}$ for the less doped ones. Thus, contrary to $T^{*}$, the doping dependence of $T_{0}$ is rather well-defined, with $T_{0}$ decreasing roughly linearly with $n_{\mathrm{h}}$, z.e. in a way opposite to $T_{c}$. As far as we can see. two different crossover temperatures now emerge from NMR measurements, with $T_{0} \geq T^{*} \geq T_{\mathrm{c}}$. Whereas the upper concentration delimiting the 
pseudogap phase at $\mathbf{Q}_{\mathrm{AF}}$ seems to coincide precisely with the optimal doping, some ambiguity remains concerning the pseudogap at $q=0$. It must be pointed out that if up to now, the opening of a pseudogap at $\mathbf{Q}_{\mathrm{AF}}$ has not been clearly observed in compounds having a single $\mathrm{CuO}_{2}$ plane per unit cell, the pseudogap behavior at $q=0$ has to be considered as a property which takes place within a single $\mathrm{CuO}_{2}$ plane. Not only it is observed in $\mathrm{La}_{2-x} \mathrm{Sr}_{x} \mathrm{CuO}_{4}$, but in the case of $(\mathrm{Bi}-\mathrm{Pb})_{2} \mathrm{Sr}_{2} \mathrm{Ca}_{2} \mathrm{Cu}_{3} \mathrm{O}_{10}$ [123] and (Tl-Pb) $\mathrm{Sr}_{2} \mathrm{Ca}_{2} \mathrm{Cu}_{3} \mathrm{O}_{9-\delta}$ [124] the oxygen MHS has been observed to decrease with the temperature for the inner plane, whereas it remains constant for the outer ones, thus showing a difference of doping for the inner and the outer planes.

It was pointed quite early that the in-plane resistivity $\rho_{\mathrm{ab}}$ also showed related anomálies, namely in optimal compounds the resistivity decreases linearly with temperature down to $T_{c}$, whereas it deviates from this linearity in all underdoped compounds, below some characteristic temperature $T_{\rho}$ (at the same time $\rho_{c}$ tends to diverge exponentially). It turns out that a majority of data indicate that $T_{\rho}=T_{0}[125,126]$, within experimental accuracy, the notable exception being $\mathrm{YBa}_{2} \mathrm{Cu}_{4} \mathrm{O}_{8}[127]$. Furthermore, a striking resemblance between $\rho_{a b} / T$ and $\chi_{0}$ was evident. Recently, both quantities have been measured in very similar single crystals of $\mathrm{HgBa}_{2} \mathrm{Ca}_{2} \mathrm{Cu}_{3} \mathrm{O}_{8+\delta}$ [29], and have been shown to scale for all temperatures almost down to $T_{\mathrm{c}}$. For now, the Hall effect and specific heat anomalies seem to correlate quite well with this pseudogap features at $q=0[126,128]$. Finally, the most spectacular evidence for the occurring of a gap above $T_{c}$ was recently given by photoemission experiments in $\mathrm{Bi}_{2} \mathrm{Sr}_{2} \mathrm{CaCu}_{2} \mathrm{O}_{8}$ [10]. These experiments have clearly shown that the Fermı surface is quite different in overdoped and underdoped compounds, with the existence of an anisotropic gap (which seems to have a d-like symmetry) already preformed above $T_{\mathrm{c}}$. This is perfectly in agreement with the NMR experiments of Walstedt et al. in $\mathrm{Bi}_{2} \mathrm{Sr}_{2} \mathrm{CaCu}_{2} \mathrm{O}_{8}$ [63].

In strongly correlated systems, the study of impurity doping effects can usually provide essential information. Particularly, the substitution of $\mathrm{Cu}$ by $\mathrm{Zn}$ in the $\mathrm{CuO}_{2}$ planes has attracted considerable attention by virtue of its dramatic effects on both the critical temperature and the AF fluctuations. The impact on the spin-gap behaviour may be summarized as follows: ${ }^{63} T_{1}$ measurements at sites far from the $\mathrm{Zn}$ impurity have shown that $T^{*}$ disappears for $\mathrm{Zn}$ concentrations as low as $1 \%$ [129], while $T_{0}$ appears to be practically insensitive to the $\mathrm{Zn}$ content from both the NMR shift [130] and resistivity measurements [131].

The pseudo spin-gap behaviour has given rise to a huge amount of theoretical work, of which we can only give a very limited and incomplete account. One of the early theoretical work explicitly tryıng to explain the NMR and INS findings at $\mathbf{Q}_{\mathrm{AF}}$ was that of Bulut and Scalapino [132], based on a dynamical nesting in the framework of the Hubbard model. In this model, the value of the gap is closely related to the difference in chemical potential with respect to the case of the half filled band. However, it is now quite clear that a drastic change in the electronic properties occurs in the vicinity of the optimal doping, which cannot be accounted in that kind of models. The fact that the distinction between underdoped and overdoped compounds, which originally described the variation of $T_{\mathrm{c}}$, extends to the metallic state of the cuprates is a strong indication that electronic correlations play a predominant role in the pairıng mechanısm. Furthermore, this suggests that the variation of $T_{\mathrm{c}}$ with doping arises from the interplay between two distinct parameters, and not from the continuous variation of a single one. In other words, there is no symmetry with respect to the optimal doping, which suggests that the two transition lines $\left(T_{\mathrm{c}}\right.$ versus $\left.n_{\mathrm{h}}\right)$ for underdoped and overdoped regime have a different orıgin (Fig. 1). This kind of behaviour is explicitly incorporated in some theoretical phase diagrams. One class is the $t$ - $J$ model treated in the framework of spin-charge separation, where the spin-gap corresponds to the formation of an RVB state [133]. In other scenarios, the spin-gap is rather the manifestation of a quantum disordered ground state, and 
the crossover from underdoped to overdoped corresponds to overdamping of localized spinexcitations $[101,105,134]$. All these approaches promote the formation of a spin-liquid state at temperatures higher than $T_{c}$ in the underdoped regime, instead of a Fermi liquid in the overdoped regime. There are also other models, such as a spin density wave gap of a metal near an AF instability $[3,135]$, the duality between itinerant and localized magnetism $[134,136]$, or peculiar dynamics related to frustrated phase separation $[84,137]$.

An intense subject of debate has concerned the proposition of Millis and Monien [122] that the spin-gap at $\mathbf{Q}_{\mathrm{AF}}$ could be related to an interplane singlet [138] pairing since its existence had only been demonstrated in bi-layer compounds at that time. Especially, no clear sign of spin-gap could be found in the single-layer $\mathrm{La}_{2-x} \mathrm{Sr}_{x} \mathrm{CuO}_{4}$. However, the objection has been raised [29] that the identical $T$-dependence of ${ }^{63} T_{1}$ in the three $\mathrm{CuO}_{2}$ planes of underdoped $\mathrm{HgBa}_{2} \mathrm{Ca}_{2} \mathrm{Cu}_{3} \mathrm{O}_{8+\delta}$ was hard to explain within an interlayer pairing model, because in such a case the inner layer should be more sensitive to the pairing. Recent investigations of the bi-layer $\mathrm{t}-\mathrm{J}$ model have also questioned the interplane singlet order [139]. Also, Wen and Lee [140] have recognized the possibility that the spin gap phase could be more stable with respect to in-plane gauge fluctuations with an $\mathrm{SU}(2)$ symmetry than with $\mathrm{U}(1)$, since this approach permits to start with a better mean field solution. One possibility to explain the apparent absence of pseudogap at $\mathbf{Q}_{\mathrm{AF}}$ in $\mathrm{La}_{2-x} \mathrm{Sr}_{x} \mathrm{CuO}_{4}$ would then be that $T^{*}$ is very close to $T_{\mathrm{c}}$ because $T_{\mathrm{c}}$ is low. On the contrary, a pseudogap at $q=0$ is unambiguously observed in this compound.

Aside the theories which explicitly include AF fluctuations, other classes of model have been proposed to explain the spin-gap as the formation of bi-polarons [141]. It has also been proposed that the underdoped regime corresponds to the existence of preformed pairs above $T_{\mathrm{c}}$ without phase coherence [142].

Although the general tendency in the NMR literature is to favour the models including AF fluctuations. the mechanism of the spin-gap formation is still a quite open question. The recent photoemission results which explicitly demonstrate that this gap is anisotropic, and that the "credo" in a large Fermi surface in all the cuprates has to be revised, is a strong stimulation to reconsider some aspects of the spin dynamics.

\section{NMR versus Inelastic Neutron Scattering}

Inelastic neutron scattering (INS) experiments can in principle directly measure the dynamical structure factor $S(\mathbf{q}, \omega) \propto \chi^{\prime \prime}(\mathbf{q}, \omega) /\left[1-\exp \left(-\hbar \omega / k_{\mathrm{B}} T\right)\right]$ as a function of $\mathbf{q}$ and $\omega$. It is thus a tool of major importance in study of the dynamic magnetic susceptibility in the cuprates. In practice, the exploration range in energy is limited between $1 \mathrm{meV}$ and $60 \mathrm{meV}$ (300 meV using spallation sources). In $q$ space, however, one cannot approach the $q=0$ region of the Brilloum zone. Moreover, the sensitivity requires large single crystals, which up to now has limited the investigations to two systems, $\mathrm{YBa}_{2} \mathrm{Cu}_{3} \mathrm{O}_{6+x}$ and $\mathrm{La}_{2-x} \mathrm{Sr}_{x} \mathrm{CuO}_{4}$. A crucial problem, when using unpolarized neutrons, is to separate the contribution of the phonons from that of the spin excitations. This difficulty can be avoided by using spin polarized neutrons, but in that case, one has to pay with a drastic reduction of the beam intensity. Obviously, any theory that claims to explain the NMR results should be consistent with the INS findings too, unless some new characteristic energy falls between a few $\mathrm{meV}$ and zero. To cover the whole literature on INS data and interpretation is beyond the scope of this paper; the reader is referred to recent reviews on the subject [143-145]. We shall only summarize the INS results which are avallable at the moment in the two systems mentioned above, before comparing them with NMR results. In both systems, evidence for antiferromagnetic fluctuations has been found in the whole metallic range. Although this point is still controversial in optimal and overdoped $\mathrm{YBa}_{2} \mathrm{Cu}_{3} \mathrm{O}_{6+x}$, recent measurements in overdoped compounds have finally confirmed the observation of magnetic 
scattering at $Q_{\mathrm{AF}}$ at low energy $\left(\leq 10 \mathrm{meV}\right.$ ) above $T_{\mathrm{c}}$ [146]. The situation is, however, quite different for the two systems as far as the shape and the temperature dependence of $\chi$ " $(q, \omega)$ is concerned. In the case of $\mathrm{La}_{2-x} \mathrm{Sr}_{x} \mathrm{CuO}_{4}$, in the low energy range $1-40 \mathrm{meV}$, the shape of $\chi^{\prime \prime}(q, \omega)$ consists of four peaks centered at $(\pi / a(1 \pm \delta), \pi / a)$ and $(\pi / a, \pi / a(1 \pm \delta))$ with $\delta=0.245 \pm 0.004$ for $x=0.14[147,148]$. The inverse length scale $\kappa$ measuring the sharpness of the peaks was found experimentally to vary as: $\kappa^{2}(T, \omega)=\kappa_{0}^{2}+a_{0}^{-2}\left(k_{\mathrm{B}} T / E_{\mathrm{T}}\right)^{2}+\left(\hbar \omega / E_{\omega}\right)^{2}$ with $\kappa_{0}=0.034 \AA^{-1}, a_{0}^{-1}=0.26 \AA^{-1}, E_{\mathrm{T}}=E_{\omega}=47 \mathrm{meV}$ [149]. These results suggest that a spin pseudogap does not open above $T_{c}$. In the case of $\mathrm{YBa}_{2} \mathrm{Cu}_{3} \mathrm{O}_{6+x}, \chi "(q, \omega)$ is peaked at $(\pi / a, \pi / a)$ for all concentrations studied. In contrast with $\mathrm{La}_{2-x} \mathrm{Sr}_{x} \mathrm{CuO}_{4}, \kappa$ was found to be independent of $T$ and $\omega$ (if one excludes the so-called "resonance" [143,144], which appears only in the superconducting state) and equal to $0.12 \AA^{-1}$ and $0.24 \AA^{-1}$ for underdoped samples belonging to the " $60 \mathrm{~K}$ " and "90 K" plateau, respectively. This result is in total disagreement with the NAFL model, as well as with the description within the quantum critical regime. A pseudo spin-gap, z.e., a transfer from low energy to high energy in the spectral weight of the spin excitations, has been clearly observed in $\mathrm{YBa}_{2} \mathrm{Cu}_{3} \mathrm{O}_{6.69}$ [143], $\mathrm{YBa}_{2} \mathrm{Cu}_{3} \mathrm{O}_{6.6}$ [150] and with difficulty in $\mathrm{YBa}_{2} \mathrm{Cu}_{3} \mathrm{O}_{6}$ 92. In the latter case, the same sample was studied by $\mathrm{NMR}$ and INS. The comparison of the temperature dependence of ${ }^{63}\left(T_{1} T\right)^{-1}$ with that of $\chi^{\prime \prime}\left(\mathbf{Q}_{\mathrm{AF}}\right.$, $\hbar \omega=10 \mathrm{meV}$ ) shows a good agreement $[85,86,116]$, although the error bars for the INS data are rather large. As already mentioned, for doping level where the INS intensity becomes too weak, the comparison between ${ }^{63}\left(T_{1} T\right)^{-1}$ and ${ }^{63}\left(1 / T_{2 \mathrm{G}}\right)$ becomes more efficient to detect the opening of a pseudogap around $\mathbf{Q}_{\mathrm{AF}}$.

Quantitative comparison between NMR and INS results can be carried out in the following way. In the case of $\mathrm{YBa}_{2} \mathrm{Cu}_{3} \mathrm{O}_{6.92}$ where the absolute amplitude of $\chi^{\prime \prime}\left(\mathbf{Q}_{\mathrm{AF}}, \hbar \omega=10 \mathrm{meV}\right)$ is not known, the following analysis has been used [85,121]. In equation (15), we make the assumption for the $q$-dependence of $\chi\left(\mathbf{Q}_{\mathrm{AF}}+q\right)$ and $\Gamma\left(\mathbf{Q}_{\mathrm{AF}}+q\right)$ that $g=f^{-1}$ (this relation occurs for the MMP susceptibility), with $f$ being either a Lorentzian or a Gaussian. It is then possible through equations $(7,10,11)$ to calculate $\Gamma_{\mathrm{AF}}$ and $\chi\left(\mathbf{Q}_{\mathrm{AF}}\right)$ as a function of $\xi$ for a given experimental set of $\left[{ }^{63}\left(T_{1} T\right)^{-1},{ }^{63} T_{2 \mathrm{G}}^{-1}\right]$ and for a given temperature (e.g. [121] $200 \mathrm{~K}$ ) above $T^{*}$. Comparison of the $\Gamma_{\mathrm{AF}}$ value with the value of $\Gamma_{\mathrm{N}}\left(Q_{\mathrm{AF}}\right)=$ $\left[2 \int_{0}^{\infty} \chi "\left(\mathbf{Q}_{\mathrm{AF}}, \omega\right) / \omega \mathrm{d} \omega\right] /\left[\pi \chi "\left(\mathbf{Q}_{\mathrm{AF}}, \omega_{\mathrm{n}}\right) / \omega_{\mathrm{n}}\right]$ deduced from the neutron experimental data on the same single crystal and at the same temperature provides a good agreement between $\Gamma_{\mathrm{N}}\left(\mathrm{Q}_{\mathrm{AF}}\right)$ and the value of $\Gamma_{\mathrm{AF}}$ obtained from the NMR data corresponding to $\kappa=0.24 \AA^{-1}$, which is precisely the value obtained from the neutron data. Thus, the INS data and the $\mathrm{Cu}$ NMR data obtained on the same sample can be interpreted quantitatively with the same parameters. However, use of the same dynamic susceptibility to calculate NSLRR at the planar oxygen site leads to a striking disagreement [121]. The calculated contribution of the AF fluctuations for $\xi / a=1$ is as large as $90 \%$ of the experimental value for a Gaussian, and $200 \%$ for a Lorentzian (see Fig. 13). In these conditions, the temperature dependence of the oxygen NSLRR should have the same behavior as the Cu NSLRR, a situation which is never observed. The conclusion is thus that either the $q$-dependence of $\lambda^{\prime \prime}(\mathbf{q}, \omega)$ at the NMR energy scale is different from that obtained at $10 \mathrm{meV}$, which is possıble, but unlıkely, or that the Mila-Rice Hamiltonian and the single spin fluid picture, including an arbitrary separation between $\chi_{Q P}^{\prime \prime}$ and $\chi_{\mathrm{AF}}^{\prime \prime}$ is inadequate to describe the dynamic part of the NMR data. The same conclusion has been reached by Walstedt et al. [151] using their NMR data and the INS data of Aeppli et al. in $\mathrm{La}_{1.85} \mathrm{Sr}_{0.15} \mathrm{CuO}_{4}$ [149]. In that case, $\chi$ " $(\mathbf{q}, \omega)$ peaks around $\mathbf{Q}_{\mathrm{AF}}$ are narrow and temperature dependent, as expected in the NAFL theories, but they are located at incommensurate positions. From INS determination of the absolute intensity of $\chi$ "(q, $\omega)$, Walstedt and 


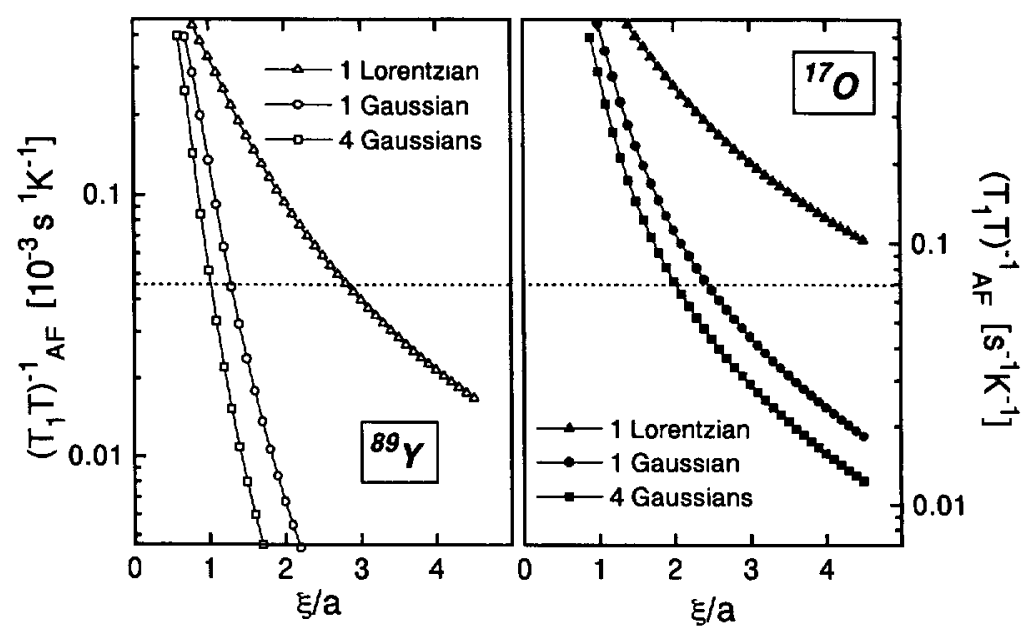

Fig. 13. - Calculated contribution of AF fluctuations to $\left(T_{1} T\right)^{-1}$ for ${ }^{89} \mathrm{Y}$ relaxation (left panel) and planar ${ }^{17} \mathrm{O}$ relaxation (right panel), as a function of the $\mathrm{AF}$ correlation length $\xi / a$ [121]. The dynamıc susceptibility at $\mathbf{Q}_{\mathrm{AF}}$ is supposed to be described by either a Lorentzian, a Gaussian or the sum of four Gaussians and adjusted to fit the $\mathrm{Cu}\left(T_{1} T\right)^{-1}$ and $\left(T_{2 \mathrm{G}}\right)^{-1}$ values in $\mathrm{YBa}_{1}{ }_{93} \mathrm{Sr}_{0}{ }_{07} \mathrm{Cu}_{3} \mathrm{O}_{6} 92$ at $200 \mathrm{~K}$. The full span of the vertical axes covers $2-200 \%$ of the experımental value of ${ }^{89} \mathrm{Y}$ and ${ }^{17} \mathrm{O}\left(T_{1} T\right)^{-1}$ at $200 \mathrm{~K}$, and the horizontal dotted line indicates $20 \%$ of it, a value indicating an approximate limit above which the AF contribution should be experimentally observable. From neutron data, $\xi / a \approx 1$.

Aeppli have concluded the NMR and the INS data could be reconcled as far as the Cu data were concerned. but again, they have shown that the calculated contribution at the oxygen site due to the AF fluctuations is too strong compared to the experimental value of ${ }^{17}\left(T_{1} T\right)^{-1}$, and they have questioned the validity of the single band picture.

At this stage, it is useful to return to the problem of the planar oxygen NSLRR. As we have seen above, for underdoped compounds, ${ }^{17} K(T)^{17}\left(T_{1} T\right)^{-1}=$ const. within a rather wide range of temperature. This can be well accounted by assuming a perfect cancellation of the AF fluctuations contributions to the NSLRR, and writing:

$$
\begin{aligned}
{ }^{17}\left(T_{1} T\right)_{\alpha}^{-1} & ={ }^{17} \gamma^{2}\left[\frac{\left(C_{\beta \beta}\right)^{2}}{\left(g_{\beta \beta}\right)^{2}}+\frac{\left(C_{\gamma \gamma}\right)^{2}}{\left(g_{\gamma \gamma}\right)}\right] \frac{k_{\mathrm{B}} \pi \chi_{0}(T)}{\left(\mu_{\mathrm{B}}\right)^{2} \Gamma_{0}} \\
& ={ }^{17} \gamma^{2}\left[\frac{K_{\beta \beta}(T) C_{\beta \beta}}{g_{\beta \beta}}+\frac{K_{\gamma \gamma}(T) C_{\gamma \gamma}}{g_{\gamma \gamma}}\right] \frac{\pi k_{\mathrm{B}}}{\mu_{\mathrm{B}} \Gamma_{0}}
\end{aligned}
$$

The value of $\hbar \Gamma_{0}$ is $0.24 \mathrm{eV}$ in $\mathrm{YBa}_{2} \mathrm{Cu}_{3} \mathrm{O}_{652}, \mathrm{YBa}_{2} \mathrm{Cu}_{3} \mathrm{O}_{7}$ [152] and $0.3 \mathrm{eV}$ in $\mathrm{La}_{1.85} \mathrm{Sr}_{0.15}$ $\mathrm{CuO}_{4}$. These are strikingly simlar results for the three compounds, in spite of quite different behavior of $\chi^{\prime \prime}(\mathbf{q}, \omega)$ as evidenced by Cu NSLRR and INS results. These features seem to indicate that in all cases the AF fluctuations do not contribute to the NSLRR of the planar oxygen, suggesting a common orıgin for ${ }^{17} T_{1}$ in all cuprates, nearly independent of the doping level. This is also supported by the absence of a field dependence of the NSLRR in the planar oxygen in $\mathrm{Tl}_{2} \mathrm{Ba}_{2} \mathrm{CuO}_{6+\delta}$, whereas the $\mathrm{Cu}$ NSLRR exhibit a logarithmic dependence as a function of $H$, which is a signature of $2 \mathrm{D}$ diffusive motion of some type of excitation. As already mentioned, there is another puzzling feature concerning the NSLRR of planar oxygen, that is the temperature dependence of the amsotropy of the NSLRR in the normal state, which 
cannot be explained simply with the usual single spin fluid picture, the Mila Rice Hamltonian, and the separation of the susceptibility into $\chi_{\mathrm{QP}}$ and $\chi_{\mathrm{AF}}$ (both of them acting on the two sites).

To solve the contradiction between the NMR and INS data as far as the oxygen NSLRR is concerned, Varma [153] recently proposed a completely different approach of the NSLRR in the $\mathrm{CuO}_{2}$ plane, based on orbital relaxation for $\mathrm{Cu}$ and oxygen sites. However, it seems difficult to completely forget the existence of the AF fluctuations. It has also been suggested that the incommensurate peaks measured by INS in $\mathrm{La}_{2}{ }_{-x} \mathrm{Sr}_{x} \mathrm{CuO}_{4}$ could be due to discommensurations rather than to the band structure. This leads to a much better filternng of the AF fluctuations at the oxygen site. since in that case the fluctuations measured locally by NMR are commensurate [154]. The same argument may also apply to YBCO, although the existence of incommensurate peaks is not settled down [150]. In a more conservative approach, Zha et al. [66] have recently proposed that the oxygen nuclei would be coupled not only to their $\mathrm{Cu}$ nearest neighbors, but also to the next nearest $\mathrm{Cu}$ neıghbors. In this way, they manage to reduce considerably the contribution of the AF fluctuations to the oxygen NSLRR. Although this possibility cannot be excluded, it seems to us that the inconsistency between INS and oxygen NMR indicates that something more fundamental has not been taken into account in the analysis currently applied to the NMR data. It is also clear that as long as the mechanism of oxygen NSLRR is not well understood, the analysis of ${ }^{63}\left(T_{1} T\right) /{ }^{63}\left(T_{2 \mathrm{G}}\right)^{z}$, where $z$ is the dynamical critical exponent, has to be considered with caution.

\section{NMR and Phase Separation}

So far, we have not mentioned the works dedicated to the possibility of phase separation between the AF background of localized spins and mobile holes. In spite of growing evidence that such a phenomenon may take place in $\mathrm{La}_{2-x} \mathrm{Sr}_{x} \mathrm{CuO}_{4+\delta}[155-157]$ and that it may even be a key feature of the physics of the cuprates [158], a possible extension of these ideas to other systems and especially to their normal state remains unproved. There have been only a few NMR/NQR studies fully devoted to this problem in the metallic phase, among which we must cite the comprehensive contribution of Teplov and collaborators [159]. We shall not further develop this point, which we feel being rising. It is clear that any kind of frustrated (dynamic) phase separation [84] will lead to averaged properties at the NMR timescale (single line, single $T_{1}$ ). but it still remains tempting to interpret some peculiar aspects of the spin dynamics discussed in this paper as connected to these inhomogeneous phases.

\section{Concluding Remarks}

One of the major difficulties in the understanding of the properties of the cuprate HTSC, is to separate what is really generic for this class of compounds, from the numerous pecularities due to the various types of blocks allowing the doping of the $\mathrm{CuO}_{2}$ planes. At the same time, it seems necessary to understand the evolution of physical properties in the whole range of doping level. So, one of the strength of NMR has been its ability to investigate most of the HTSC cuprate famlies. at varıous doping levels, and to reveal very early some quite general features: 1) The existence of antiferromagnetic fluctuations in the whole range of doping of the HTSC has been revealed by numerous experiments. The ratio of the $\mathrm{Cu}$ nuclear spinlattice relaxation rate to that of the planar oxygen is a monotonously increasing function with decreasing $T$. The indirect nuclear spin-spin interaction ( $T_{2 G}$ measurements on copper) is also continuously increasing with decreasing $T$ down to $T_{\mathrm{c}}$, showing the temperature dependence of the staggered susceptibility $\chi\left(\mathbf{Q}_{\mathrm{AF}}\right)$. It is clear that these temperature dependences weaken 
as the doping level is increased, however, they are still present even in the overdoped regime. Perhaps one of the most spectacular evidence for the unusual magnetic susceptibility in the normal state of the cuprates has been revealed by the observation of local magnetic moment induced by a non-magnetic impurity in the $\mathrm{CuO}_{2}$ plane [130].

ii) The striking difference between the magnetic properties of the underdoped and the overdoped compounds was first revealed by NMR through two phenomena: One is the decrease of the static spin susceptibility below some temperature $T_{0}$, which was finally recognized as due to the opening of a pseudogap, observed as well on spin and charge properties for all underdoped compounds. Another one is a maximum in ${ }^{\mathrm{Cu}}\left(T_{1} T\right)^{-1}$ at some temperature $T^{*},\left(T_{\mathrm{c}}<T^{*}<T_{0}\right)$, followed by a simultaneous decrease of ${ }^{\mathrm{Cu}}\left(T_{1} T\right)^{-1}$ and an increase of $\mathrm{Cu}^{\mathrm{C}}\left(T_{2 \mathrm{G}}\right)^{-1}$ with decreasing temperature, which is the signature of a pseudogap for the spinexcitations at $\mathbf{Q}_{\mathrm{AF}}$. This pseudogap is present in the whole underdoped regime, and disappears precisely at the optimal doping. However, it has not been convincingly observed the single-layer material $\mathrm{La}_{2-x} \mathrm{Sr}_{x} \mathrm{CuO}_{4}$, so the question whether its opening is due to the coupling between adjacent $\mathrm{CuO}_{2}$ layers or is an intrinsic property of a single layer is still a controversial point.

The origin of these pseudogaps, the difference between most of the physical properties in the underdoped and overdoped regimes, like resistivity, Hall effect, specific heat or photoemission, are presently the subject of an intense debate.

Quantitative interpretation of NMR data requires a model for the dynamıc spin susceptibility $\chi$ " $(\mathbf{q}, \omega)$, and therefore is more subject to controversy. However. it comes out from analysis of $T_{1}$ and $T_{2 \mathrm{G}}$ that the energy scale $\Gamma_{\mathrm{AF}}$ of the antiferromagnetic fluctuations, is larger in the systems where the optimal $T_{\mathrm{c}}$ is the highest. This is usually accompanied by a larger value of the supertranferred hyperfine field $B$ between nearest neighbors copper, and a smaller value of the quadrupolar coupling constant $\nu_{\mathrm{Q}}$ for the planar copper. A comparison between NMR and inelastic neutron scattering results is obviously of primary importance to test the various models of $\chi^{\prime \prime}(\mathbf{q}, \omega)$. Several models refer to commensurate AF fluctuations $\chi$ " $(\mathbf{q}, \omega)$ peaked at

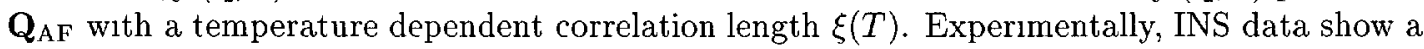
temperature dependence for $\xi$, but incommensurate peaks in $\mathrm{La}_{2-x} \mathrm{Sr}_{x} \mathrm{CuO}$, and conversely, $T$-independent $\xi$ and commensurate fluctuations in $\mathrm{YBa}_{2} \mathrm{Cu}_{3} \mathrm{O}_{6+x}$. Using the neutron data on $\chi^{\prime \prime}(\mathbf{q}, \omega)$ to interpret the NMR relaxation in both systems leads to a quantitative agreement as far as the copper nuclear spin-lattice relaxation is concerned, but to a severe failure in the case of the planar oxygen, for which the contribution of the AF fluctuations is found experimentally much less than the computed one. Since it is unlikely that there exists another characteristic energy between the meV and the nuclear Zeeman energy $(\approx \mu \mathrm{eV})$, it is clear that we have to revise, at least partially, our way of thinking the nuclear spin-lattice relaxation in the cuprates, perhaps including a better microscopic description of the hole dynamics in the $\mathrm{CuO}_{2}$ planes.

\section{Note Added in Proofs}

In a recent preprint, $\mathrm{Y}$. Itoh et al. show by ${ }^{63} \mathrm{Cu}$ NMR the existence of a spin pseudogap at $Q_{\mathrm{AF}}$ in the underdoped, single layered, $\mathrm{HgBa}_{2} \mathrm{CuO}_{4+\delta}$. In the heavily doped regime $\left(T_{\mathrm{c}}=50 \mathrm{~K}\right)$, they found $T^{*} \sim 250 \mathrm{~K}$, which is comparable to the value found in underdoped $\mathrm{HgBa}_{2} \mathrm{Ca}_{2} \mathrm{Cu}_{3} \mathrm{O}_{8+\delta}$ with $T_{\mathrm{c}}=115 \mathrm{~K}$.

\section{Acknowledgments}

The authors would like to thank Dr. P. Carretta for fruitful discussions and Professor W.G. Clark for a critical reading of the manuscript. 


\section{References}

[1] Bednorz J.G. and Müller K.A., Z. Physik B64 (1987) 189-193.

[2] Hirsh J.E. and Scalapino D.J., Phys. Rev. Lett. 56 (1986) 2732-2735; Labbé J. and Bok J., Europhys. Lett. 3 (1987) 1225-1230; Friedel J., J. Phys. Condens. Matter 1 (1989) $7757-7762$.

[3] Kampf A. and Schrieffer J.R., Phys. Rev. B 41 (1990) 6399-6408.

[4] Montoux P. and Pines D., Phys. Rev. B 49 (1994) 4261; Pines D. and Montoux P., J. Phys. Chem. Solzds 56 (1995) 1651-1658 and references therem.

[5] Anderson P.W., Science 235 (1987) 1196; in "Frontiers and Borderlines in Many Particle Physics, Proceedings of the Varenna Summer Shool, Varenna, Italy, 1987", R.A. Broglia and J.R. Schrieffer Eds. (North Holland, Amsterdam, 1988).

[6] Physica C 235-240 (Proceedings of 4th M2HTSC, Grenoble 1994, P. Wyder Ed.).

[7] It is not clear if electron doping generates the same physics as hole doping. Therefore, we shall only consider the case of hole doping, on which much of the experimental activity has been concentrated. For NMR investigations of hole doped cuprates see Ima1 T., Slichter C.P., Cobb J.L. and Markert J.T., J. Phys. Chem. Solzds 56 (1995) 1921-1925, and references therein.

[8] Graf T., Triscone J.-M. and Muller J., J. Less Common Metals 159 (1990) 349; Genoux J.Y. , Graf T., Junod A., Sanchez D., Triscone J.-M. and Muller J., Physıca C 177 (1991) 315-321.

[9] Takag1 H., Cava R.J., Marezio M., Batlogg B., Krajewski J.J., Peck W.F., Jr., Bordet P. and Cox D.E., Phys. Rev. Lett. 68 (1992) 3777-3780.

[10] Marshall D.S., Dessau D.S., Loeser A.G., Park C.-H., Matsuura A.Y., Eckstein J.N., Bosovic I., Fournier P., Kapitulnik A., Spicer W.E. and Shen Z.-X., Phys. Rev. Lett. 76 (1996) 4841-4844.

[11] Fukuzumı Y., Mizuhashi K. Takenaka K. and Ushida S., Phys. Rev. Lett. 76 (1996) 684687.

[12] Ando Y., Boebinger G. S., Passner A., Kimura T. and Kishio K.. Phys. Rev. Lett. 75 (1995) 4662-4665.

[13] Rice T.M., in "Physics and Chemistry of Oxide Superconductors", Y. Iye and H. Yasuoka Eds. (Springer-Verlag. Berlin, 1989) pp. 313-322.

[14] Berthier C., Berthier Y., Butaud P., Clark W.G., Gillet J.A., Horvatić M., Ségransan P. and Henry J.Y., Appl. Magn. Reson. 3 (1992) 449-468.

[15] Hebel L.C. and Slichter C.P., Phys. Rev. 107 (1957) 901-908.

[16] MacLaughlin D.E., in "Solid State Physics", vol. 31, Seitz and Türnbull Eds. (Accadem1c Press, New York, 1976) p. 1-69.

[17] Pennington C.H. and Slichter C.P., in "Pysical Properties of high temperature superconductors", vol II. D.M. Ginsberg Ed. (World Scientific, New Jersey, 1990).

[18] Mehring M. (guest editor), Appl. Magn. Reson. 3 (1992).

[19] Brinkmann D. and Mali M., in "NMR Basıc Principles and Progress' , Vol. 31 (Springer Verlag, Berlin. Heidelberg 1994) pp. 171-211.

[20] Slichter C.P., in "Stongly Correlated Electronic Systems", K.S. Bedell et al. Eds. (AddisonWesley, New York, 1994).

[21] Borsa F., Carretta P.. Cintolesı F.. Corti M., Rigamonti A.. Suh B.J. and Torgeson D., Appl. Magn. Reson. 9 (1995) 149-164.

[22] Asayama K., Kitaoka Y., Zheng G.-Q. and Ishida K., Prog. NMR Spectrosc. 28 (1996) 221-253. 
[23] Walstedt R.E., Warren W.W. Jr., Bell R.F., Brennert G.F., Espinosa G.F., Remeika J.P., Cava R.J. and Rietman E.A., Phys. Rev. B 36 (1987) 5727-5730.

[24] Mali M., Brinkmann D., Pauli L.. Roos J. and Zimmermann H., Phys. Lett. A 124 (1987) 112-116.

[25] Farrell D.E., Chandrasekhar B.S., DeGuire M.R., Fang M.M., Kogan V.G., Clem J.R. and Finnemore D.K., Phys. Rev. B 36 (1987) 4025-4027.

[26] Vega A.J., Farneth W.E., McCarron E.M. and Bordia R.K., Phys. Rev. $B 39$ (1989) 2279-2332.

[27] Lütgemeier H., Heinmaa I. and Egorov A.V.. Phys. Scri. T49 (1993) 137-142.

[28] Zheng G.. Kitaoka Y., Ishida K. and Asayama K., J. Phys. Soc. Jpn 64 (1995) 2524-2532.

[29] Julien M.H., Horvatić M., Carretta P., Berthier C.. Berthier Y., Ségransan P., Carrıngton A. and Colson D., Phys. Rev. Lett. 76 (1996) 4238-4240.

[30] Magishi K-I., Kitaoka Y.. Zheng G-Q., Asayama K., Tokiwa K., Iyo A. and Ihara H., J. Phys. Soc. Jpn 64 (1995) 4561-4565.

[31] Shimizu T., J. Phys. Soc. Jpn 62 (1993) 779-884.

[32] Winter N.W., Merzbacher C.I. and Violet C.E., Appl. Spectroscop. Rev. 28 (1993) 123-64.

[33] In-plane anisotropy is beyond experimental resolution.

[34] Note that the hyperfine field is often given in kGauss per $\mu_{\mathrm{B}}$; in that case, the value is divided by the $g$-factor.

[35] Narath A., in "Hyperfine Interactions", A.J. Freeman and R.B. Frankel Eds. (Academic Press, 1967).

[36] Winter J., Magnetic resonane in metals (Clarendon Press, Oxford, 1971)/

[37] Takigawa M., Hammel P.C., Heffner R.H., Fisk Z., Smith J.L. and Schwarz R.B., Phys. Rev. B 39 (1989) 300-304.

[38] Pennıngton C.H., Durand D.J., Slichter C.P., Rice J.P., Bukowski E.D. and Ginsberg D.M., Phys. Rev. B 39 (1989) 2902-2905.

[39] Mila F. and Rice T.M., Physıca C 157 (1989) 561-570.

[40] Takigawa M., Hammel P.C., Heffner R.H., Fisk Z., Ott K.C. and Thompson J.D., Phys. Rev. Lett. 63 (1989) 1865-1868.

[41] Horvatić M., Berthier Y., Butaud P., Kitaoka Y., Ségransan P., Berthier C., KatayamaYoshida H., Okabe Y. and Takahashi T., Physica C 159 (1989) 689-696.

[42] Yoshinari Y., Yasuoka H., Ueda Y., Koga K. and Kosuge K., J. Phys. Soc. Jpn 59 (1990) 3698-3711.

[43] Alloul H., Mendels P., Collın G., and Monod P., Phys. Rev. Lett. 61 (1988) 746; Adrian F.J., Phys. Rev. Lett. 61 (1988) 2148-2151.

[44] Moriya T.. J. Phys. Soc. Jpn 18 (1963) 516-523.

[45] Barrıquand F., Odier P., Jérome D., Physica C 177 (1991) 230-235.

[46] Horvatić M., Berthier C., Berthier Y., Ségransan P., Butaud P., Clark W.G., Gillet J.A. and Henry J.Y., Phys. Rev. B 48 (1993) 13848-13864.

[47] Pennington C.H., Durand D.J., Slichter C.P.. Rice J. P., Bukowski E.D. and Ginsberg D.M., Phys. Rev. B 39 (1989) 274-277.

[48] Itoh Y., Yasuoaka H., Fujiwara Y., Ueda Y.. Machi T., Tomeno I. Tai K. Koshizuka N. and Tanaka S., J. Phys. Soc. Jpn 61 (1992) 1287-1298.

[49] Imai T., Slichter C.P., Paulıkas A.P. and Veal B.. Phys. Rev. B 47 (1993) 9158-9161.

[50] Rice T. M. and Zhang F.C., Phys. Rev. B 37 (1998) 3759-3761.

[51] Emery V. J. and Reiter G., Phys. Rev. B 38 (1988) 11938-11941.

[52] Alloul H., Ohno T. and Mendels P., Phys. Rev. Lett. 63 (1989) 1700-1703.

[53] Mila F. and Rice T.M., Phys. Rev. B 40 (1989) 11382-11385.

[54] Takigawa M., Reyes A.P., Hammel P.C., Thompson J.D., Heffner R.H., Fisk Z. and Ott 
K.C., Phys. Rev. B 43 (1991) 247-257.

[55] Mangelschots I., Ma'lı M., Roos J., Brinkmann D., Rusiecki S., Karpinski J. and Kaldis E., Physica C 194 (1992) 277-286; Mangelschots I., Mali M., Roos J., Stern R., Bankay M., Lombardi A. and Brinkmann D., in "Phase separation in cuprate superconductors", K.A. Müller and G. Benedek Eds. (1993, World Scientific, Singapore) p. 262-279.

[56] Ishida K., Kitaoka Y. Zheng G-Q. and Asayama K., J. Phys. Soc. Jpn 60 (1991) 3516-3524

[57] Clogston A.M., Jaccarino V. and Yafet K.. Phys. Rev. 133A (1964) 1630-1637.

[58] Shastry B.S., Phys. Rev. Lett. 63 (1989) 1288-1291.

[59] Shimızu T., Yasuoka H., Tsuda T., Koga K. and Ueda Y., Bull. Magn. Res. 12 (1990) 39-54.

[60] Tomeno I., Machi T., Tai K., Koshizuka N., Kambe S., Hayashi A., Ueda Y. and Yasuoka H., Phys. Rev. B 49 (1994) 15327-15334.

[61] Kambe S., Yasuoka H., Hayashi A. and Ueda Y., Phys. Rev. B 47 (1993) 2825-2834.

[62] Zheng G-Q.. Kitaoka Y., Asayama K., Hamada K., Yamauchi H., and Tanaka S.. J. Phys. Soc. Jpn 64 (1995) 3184-3187; Physica C 260 (1996) 197-210.

[63] Walstedt R.E., Bell R.F. and Mitzi D.B., Phys. Rev. B 44 (1991) 7760-7763.

[64] Ishida K., Kitaoka Y., Asayama K., Kadowaki K. and Mochiku T., Physica C 263 (1996) 371-374.

[65] Takigawa M., Hults W.L. and Smith J.L., Phys. Rev. Lett. 71 (1993) 2650-2653.

[66] Zha Y., Barzykhin V. and Pines D., Phys. Rev. B (1996) in press.

[67] Kambe S., Machi T., Tomeno I., Yasuoka H., Hayashi A. and Ueda Y., J. Phys. Soc. Jpn 63 (1994) 3481-3487.

[68] Bucci C. and Guidi G., Phys. Rev. B 9 (1974) 3053-3063.

[69] Thelen D. and Pines D., Phys. Rev. B 49 (1994) 3528-3533.

[70] Takigawa M.. Phys. Rev. B 49 (1994) 4158-61.

[71] Ohsugi S., Kitaoka Y., Ishida K. and Asayama K., J. Phys. Soc. Jpn 60 (1991) 23512360; Ohsugi S., Kitaoka Y., Ishida K., Zheng G-Q. and Asayama K., J. Phys. Soc. Jpn 63 (1994) 700-715.

[72] Imai T., Slichter C. P.. Yoshimura K. and Kosuge K., Phys. Rev. Lett. 70 (1993) 10021005.

[73] Matsumura M., Itoh Y., Yasuoka H., Ueda Y. and Yamagata H., J. Phys. Soc. Jpn 65 (1996) 699-702.

[74] Zimmermann H., Mali M., Brinkmann D., Karpinski J., Kaldis E. and Rusiecki S., Physica C 159 (1989) 681-688.

[75] Zheng G-Q., Kuse T.. Kitaoka Y., Ohsugi S., Asayama K. and Yamada Y., Physıca $C$ 208 (1993) 339-346.

[76] Yoshinari Y., Yasuoka H. and Ueda, J. Phys. Soc. Jpn 61 (1992) 770-781.

[77] Mehring M. et al. in reference [18]; Hentsch F., Winzek N., Mehring M., Mattausch Hj., Simon A. and Fermer R., Physica C 165 (1990) 485-492.

[78] Bankay M., Malı M. Roos J. and Brinkmann D., Phys. Rev. B 50 (1994) 6416-6425.

[79] Alloul H., Mahajan A., Casalta H., and Klein O.. Phys. Rev. Lett. 70 (1993) 1171-1173.

[80] Barriquand F. and Sawatzky G.A., Phys. Rev. B 50 (1994) 16649-16667.

[81] Kambe S., Yasuoka H., Hayashi A. and Ueda Y., Phys. Rev. Lett. 73 (1994) 197-200.

[82] Vyaselev O.M., Kolesnikov N.N., and Shegolev I.F., JETP Lett. 60 (1994) 824-828.

[83] Benner H. and Boucher J.P., in "Magnetic Properties of Layered Transition Metal Compounds", L.J. De Jongh Ed. (1990, Kluwer Academıc Publishers, Netherlands) pp. 323378.

[84] Emery V.J. and Kivelson S.A., Physıca C 209 (1993) 597-621; Zaanen J., Horbach M.L. and van Saarloos W., Phys. Rev. B 53 (1996) 8671-8680. 
[85] Berthier C., Gillet J.A., Auler T., Berthier Y., Horvatić M, Ségransan P. and Henry J.Y., Phys. Scrt. T 49 (1993) 131-136.

[86] Berthier C., Horvatić M, Carretta P., Berthier Y, Ségransan P., Gillet J. A., Auler T. and Henry J.Y., Physica C 235-40 (1994) 67-70.

[87] Stern R, Mali M., Roos J. and Brinkmann D., Phys. Rev. B 51 (1995) 15478-15483.

[88] Corey R.L., Curro N.J., O'Hara K., Imai T., Slichter C.P., Yoshimura K., Katoh M. and Kosuge K., Phys. Rev. B 53 (1996) 5907-5914.

[89] Itoh Y., Yasuoka H., Hayashi A. and Ueda Y., J. Phys. Soc. Jpn 63 (1994) 22-25.

[90] Imai T., Slichter C.P., Yoshimura K., Katoh K. and Kosuge K., Phys. Rev. Lett. 71 (1993) 1254-1257.

[91] Walstedt R.E. and Cheong S.W., Phys. Rev. B 51 (1995) 3163-3175; Phys. Rev. B 53 (1996) R6030-R6034.

[92] Stern R., Mali M., Mangelschots I., Roos J., Brinkmann D., Genoud J.-Y.. Graf T. and Muller J., Phys. Rev. B 50 (1994) 426-437.

[93] Stern R., Mali M., Roos J. and Brinkmann D., Phys. Rev. B 52 (1995) R15734-R15737.

[94] We recall that the nuclear spin-spin interaction is mediated by the electronc spin susceptibility in the cuprates.

[95] Millis A.J. and Monien H., preprint cond-mat/9606008.

[96] Reznik D.. Bourges P., Fong H.F., Regnault L.P.. Bossy J., Vettier C., Millius D.L., Aksay I.A. and Keimer B., Phys. Rev. B 53 (1996) R14741-R14744.

[97] Kampf A.P., Phys. Rep. 249 (1994) 219-351.

[98] Millis A.J., Monien H. and Pines D., Phys. Rev. B 42 (1990) 167-178.

[99] Millis A.J. and Monıen H., Phys. Rev. B 45 (1992) 3059-3076.

[100] Mornya T.. Takahashi Y. and Ueda K., J. Phys. Soc. Jpn 59 (1990) 2905-2915.

[101] Sokol A. and Pines D., Phys. Rev. Lett. 71 (1993) 2813-2816.

[102] Chubukov A.V. and Sachdev S., Phys. Rev. Lett. 71 (1993) 169-172.

[103] Littlewood P.B., Zaanen J., Aeppli G. and Monien H., Phys. Rev. B 48 (1993) 487-498.

[104] Monien H., Pines D. and Takigawa M., Phys. Rev. B 43 (1991) 258-274.

[105] Barzykin V. and Pines D., Phys. Rev. B 52 (1995) 13585-13600.

[106] Chakravarty S., Halperin B., Nelson D.R.. Phys. Rev. B 39 (1989) 2344-2371.

[107] Kü̈bert C. and Muramatsu M., Int. J. Mod. Phys. B (in press).

[108] The applicability of this scaling relation has been discussed in a recent preprint by Carretta $P$., Rigamonti $A$. and Sala $R$., who pointed out that in the quantum critical regime $\chi\left(Q_{\mathrm{AF}}\right)$ is proportionnal to $\xi^{1-\eta} / T$. Eventhough, their conclusions regarding $T_{1} T / T_{2 \mathrm{G}}$ remain unchanged.

[109] Using a properly chosen linear combination of $\left(T_{1} T\right)_{c}^{-1}$ and $\left(T_{1} T\right)_{a b}^{-1}$, the ratio $T_{1} T / T_{2 \mathrm{G}}$ can be made nearly independant of the values of the hyperfine coupling constants (see Ref. [105])

[110] Itoh Y. and Yasuoka H., J. Phys. Soc. Jpn 63 (1994) 2518-2521.

[111] See for instance contributions by Uchida S., Shen Z.X., Timusk T. and Loram J.W., in the Proceedings of the 10th Anniversary HTS Workshop on Physics, Materials and Applications, Houston, March 12-16, 1996 (World Scientific Publishing. to be published): see also Phys. Today (June 1996) 17-19.

[112] Warren Jr.W.W., Walstedt R.E., Brennert G.F., Cava R. J., Tycko R., Bell R.F. and Dabbagh G., Phys. Rev. Lett. 62 (1989) 1193-1196.

[113] Horvatić M., Ségransan P., Berthier C., Berthier Y., Butaud P., Henry J.Y., Couach M. and Chaminade J.P., Phys. Rev. B 39 (1989) 7322-7325.

[114] Yasuoka H., Imas T. and Shimizu T., in "Strong Correlations and Superconductivity", H. Fukuyama et al. Eds. (Springer Verlag, Berlin, 1989) pp. 254-289. 
[115] Rossat-Mignod J., Regnault, L. P., Vettier C., Bourges P.. Burlet P., Bossy J., Henry J.Y. and Lapertot G., Physıca C 185-189 (1991) 86-92.

[116] Horvatić M.. Auler T.. Berthier C., Berthier Y., Butaud P.. Clark W.G., Gillet J.A., Ségransan P. and Henry J. Y., Phys. Rev. B 47 (1993) 3461-3464.

[117] The sample studied in reference [49] is labelled as $\mathrm{YBa}_{2} \mathrm{Cu}_{3} \mathrm{O}_{6}$, but is likely to belong to the overdoped part of the $\mathrm{YBa}_{2} \mathrm{Cu}_{3} \mathrm{O}_{6+x}$ phase diagram.

[118] Goto A., Yasuoka H., Otzschi K. and Ueda Y.J., Phys. Soc. Jpn 64 (1995) 367-370.

[119] Machi T., Tomeno I., Tai K., Koshizuka N. and Yasuoka H., Physica $C 226$ (1994) $227-234$

[120] Matsumura M., Sakamoto Y.. Fushihara T.. Itoh Y. and Yamagata H., J. Phys. Soc. Jpn 64 (1995) 721-724.

[121] Gillet J.A., Auler T., Horvatić M., Berthier C., Berthier Y., Ségransan P. and Henry J.Y., Physica C 235-240 (1994) 1667-1668; Gillet J.A., Thesis (Grenoble, 1994).

[122] Millis A.J. and Monien H., Phys. Rev. Lett. 70 (1993) 2810-2813.

[123] Trokiner A., Le Noc L., Schneck J., Pougnet A.M., Mellet R., Primot J., Savary H., Gao Y.M. and Aubry S., Phys. Rev. B 44 (1991) 2426-2429; Le Noc L., Trokiner A., Schneck J., Pougnet, Morin D., Savary H., Yakubovskii A., Mykhalyov K.N. and Verkhovskii S.V., Physıca C 235-240 (1994) 1703-1704.

[124] Han Z.P., Dupree R., Howes A.P., Liu R.S. and Edwards P.P., Physıca C 235-240 (1994) 1709-1710.

[125] Ito T., Takenada K. and Uchida S., Phys. Rev. Lett. 70 (1993) 3995-3998.

[126] Batlogg B., Hwang H.Y., Takagı H., Cava R.J., Kao H.L. and Kwo J., Physica C 235-240 (1994) 130-133.

[127] Bucher B., Steiner P., Karpinski J., Kaldis E. and Wachter P., Phys. Rev. Lett. 70 (1993) 2012-2015.

[128] Loram J.W., Mirza K.A., Wade J.M., Cooper J.R. and Liang W.Y., Physica C 235-240 (1994) 134-137: Loram J.W., Mirza K.A., Cooper J.R. and Liang W.Y., Phys. Rev. Lett. 71 (1993) 1740-1743.

[129] Zheng G.-Q., Odaguchi T., Mito T., Kitaoka Y., Asayama K. and Kodama Y., J. Phys. Soc. Jpn 62 (1993) 2591-2594.

[130] Mahajan A.V., Alloul H., Collin G. and Marucco J.F., Phys. Rev. Lett. 72 (1994) 31003103; Williams G.V.M., Tallon J.L., Meinhold R. and Jánossy A., Phys. Rev. B 51 (1995) 16503-16506.

[131] Walker D.J.C., Mackenzie A.P. and Cooper J.R., Phys. Rev. B 51 (1995) 15653-15656; Mizuhashi K., Takenada K., Fukuzumı Y. and Uchida S., Phys. Rev. B 52 (1995) R3884R3887.

[132] Bulut N. and Scalapıno D.. Phys. Rev. Lett. 67 (1991) 449-452; Lavagna M. and Stemman G., Phys. Rev. B 49 (1994) 4235-4250.

[133] Tanamoto T., Kohno H. and Fukuyama H., J. Phys. Soc. Jpn 61 (1992) 1886-1894;

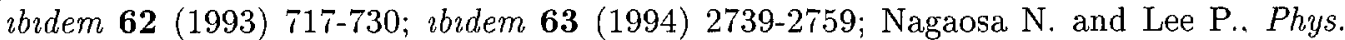
Rev. B 45 (1992) 966-980; Stemman G., Pepın C. and Lavagna M., Phys. Rev. B 50 (1994) 4075-4085.

[134] Onufrieva F. and Rossat-Mignod J., Phys. Rev. B 52 (1995) 7572-7603.

[135] Charfi-Kaddour S., Tarento R.J. and Heritier M., J. Phys. I France 2 (1992) 1853-1860.

[136] Miyake K. and Narkiyo O.. J. Phys. Soc. Jpn 63 (1994) 3821-3837; Narkiyo O. and Miyake K., J. Phys. Soc. Jpn 63 (1994) 4169-4175.

[137] Rice T.M., Troyer M. and Tsunetsugu H., J. Phys. Chem. Solids 56 (1995) 1663-1667; Tsunetsugu H., Troyer M. and Rice T.M., Phys. Rev. B 51 (1995) 16456-16459. 
[138] Altshuler B.L., Ioffe L.B. and Millis A.J., Phys. Rev. B 53 (1996) 415-424 and references therein.

[139] Eder R., Ohta Y. and Maekawa S., Phys. Rev. B 52 (1995) 7708-7712; Normand B., Kohno H. and Fukuyama H., J. Phys. Soc. Jpn 64 (1995) 3903-3915.

[140] Wen X.-G. and Lee P.A., Phys. Rev. Lett. 76 (1996) 503-506.

[141] Alexandrov A.S., Bratkovsky A.M. and Mott N.F., Phys. Rev. Lett. 72 (1994) 1734-1737; Ranninger J. and Robin J.M., Phys. Rev. B 53 (1996) R11961-R11963.

[142] Doniach S. and Invi M., Phys. Rev. B 41 (1990) 6668-6678; Randeria M., Trivedi M., Moreo A. and Scalettar R.T., Phys. Rev. Lett. 69 (1992) 2001-2004; Emery V.J. and Kivelson, Nature 374 (1995) 434-437.

[143] Rossat-Mignod J., Regnault L.P. , Bourges P., Vettier C., Burlet P. and Henry J.Y., Frontiers in Solid State Sciences Vol.1. L.C. Gupta and S. Multan Eds. (World Scientific 1993) pp. 265-348.

[144] Regnault L.P., Bourges P., Burlet P., Henry J. Y., Rossat-Mignod, Sidis Y. and Vettier C., Physica C 235-240 (1994) 59-62.

[145] Birgeneau R.J., Aharony A., Belk N.R., Chou F.C., Endoh Y., Greven M., Hosoya S., Kastner M.A., Lee C.H., Lee Y.S., Shirane G., Wakimoto S., Wells B.O. and Yamada K., J. Phys. Chem. Soluds 56 (1995) 1913-1919.

[146] Regnault L.P. and Bourges P. (private communication).

[147] Cheong S.W., Aeppli G., Mason T. E., Hayden S. M., Mook H.A., Canfield P.C., Fisk Z., Clausen K.N. and Martinez J. L., Phys. Rev. Lett. 67 (1991) 1791-1794.

[148] Mason T.E., Aeppli G. and Mook H.A., Phys. Rev. Lett. 68 (1992) 1414-1417.

[149] Aepplı G., Mason T.E., Hayden S.M. and Mook H.A., preprint.

[150] Tranquada J.M., Gehring P.M., Shirane G., Shamoto S. and Sato M., Phys. Rev. B 46 (1992) 5561-5575.

[151] Walstedt R.E., Shastry B.S., Cheong S.-W., Phys. Rev. Lett. 72 (1994) 3610-3613.

[152] Berthier C., Berthier Y., Butaud P., Clark W. G., Gillet J. A., Horvatić M., Ségransan P. and Henry J.Y., in "Electronic Properties and Mechanisms of High $T_{\mathrm{c}}$ Superconductors", T. Oguchi, Kadowaki and T. Sasaki, Eds. (Elsevier Science Publishers B.V., 1992) pp. $347-351$.

[153] Varma C.M., preprint; Varma C.M.. Phys. Rev. Lett. 75 (1995) 898-891.

[154] Barzykin V., Pines D. and Thelen D., Phys. Rev. B 50 (1994) 16052-16059.

[155] Borsa F., Carretta P., Cho J.H., Chou F.C., Hu Q., Johnston D.C., Lascialfari A., Torgeson D.R., Gooding R.J., Salem N.M. and Vos K.J.E., Phys. Rev. B 52 (1995) 73347345.

[156] Mehring M., Baehr M., Gergen P., Gross J., Kessler C. and Winzek N., in "Phase separation in cuprate superconductors", K.A. Müller and G. Benedek Eds. (World Scientific. Singapore, 1993) pp. 67-83.

[157] Statt B.W., Hammel P.C.. Fisk Z., Cheong S.W., Chou F.C., Johnston D.C. and Schirber J.E., Phys. Rev. B 52 (1995) 15575-15581.

[158] Tranquada J.M., Sternlieb B.J., Axe J.D., Nakamura Y. and Uchida S., Nature 375 (1995) 561-563.

[159] Teplov M.A., Kryukov E.V., Duglav A.V., Egorov A.V. and Mor K., JETP Lett. 63 (1996) 227-233; Bakharev O.N., Eremın M.V., and Teplov M.A., JETP Lett. 61 (1995) 515-519; Teplov M.A., Baharev O.N., Dooglav A.V., Egorov A.V., Krjukov E.V., Mukhamedshin I.R., Sakhratov Yu.A., Brom H.B., and Witteveen J., Proceedings of NATO ASI: Materials aspects of High $T_{c}$ superconductivity: 10 years after the discovery., Delphi, 19-31 August 1996, Liarokapis Ed. (Kluwer academıc publishers, Dordrecht, to be published). 